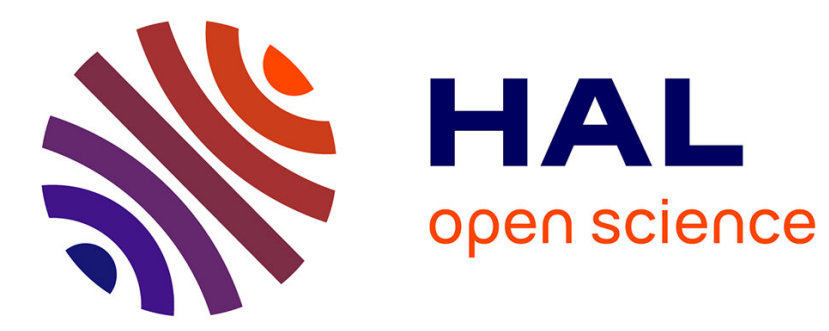

\title{
Modelling Polycrystalline Materials: An Overview of Three-Dimensional Grain-Scale Mechanical Models
}

\author{
Ivano Benedetti, Fabrice Barbe
}

\section{To cite this version:}

Ivano Benedetti, Fabrice Barbe. Modelling Polycrystalline Materials: An Overview of ThreeDimensional Grain-Scale Mechanical Models. Journal of Multiscale Modelling, 2014, 05 (01), pp.1350002. 10.1142/S1756973713500029 . hal-02327297

\section{HAL Id: hal-02327297 \\ https://hal.science/hal-02327297}

Submitted on 2 Sep 2020

HAL is a multi-disciplinary open access archive for the deposit and dissemination of scientific research documents, whether they are published or not. The documents may come from teaching and research institutions in France or abroad, or from public or private research centers.
L'archive ouverte pluridisciplinaire HAL, est destinée au dépôt et à la diffusion de documents scientifiques de niveau recherche, publiés ou non, émanant des établissements d'enseignement et de recherche français ou étrangers, des laboratoires publics ou privés. 


\title{
MODELLING POLYCRYSTALLINE MATERIALS: AN OVERVIEW OF THREE-DIMENSIONAL GRAIN-SCALE MECHANICAL MODELS.
}

\author{
I. BENEDETTI* \\ Dipartimento di Ingnegneria Civile, Ambientale, Aerospaziale, dei Materiali \\ Università degli Studi di Palermo, Viale delle Scienze, Edificio 8 \\ Palermo, 90128, Italy \\ F. BARBE \\ INSA Rouen, Groupe de Physique des Matriaux, CNRS UMR 6634 \\ Saint Étienne du Rouvray, 76801, France
}

Received (Day Month Year)

Revised (Day Month Year)

\begin{abstract}
A survey of recent contributions on three-dimensional grain-scale mechanical modelling of polycrystalline materials is given in this work. The analysis of material microstructures requires the generation of reliable micro-morphologies and affordable computational meshes as well as the description of the mechanical behavior of the elementary constituents and their interactions. The polycrystalline microstructure is characterized by the topology, morphology and crystallographic orientations of the individual grains and by the grain interfaces and microstructural defects, within the bulk grains and at the inter-granular interfaces. Their analysis has been until recently restricted to twodimensional cases, due to high computational requirements. In the last decade, however, the wider affordability of increased computational capability has promoted the development of fully three-dimensional models. In this work, different aspects involved in the grain-scale analysis of polycrystalline materials are considered. Different techniques for generating artificial micro-structures, ranging from highly idealized to experimentally based high-fidelity representations, are briefly reviewed. Structured and unstructured meshes are discussed. The main strategies for constitutive modelling of individual bulk grains and inter-granular interfaces are introduced. Some attention has also been devoted to three-dimensional multiscale approaches and some established and emerging applications have been discussed.
\end{abstract}

Keywords: Polycrystalline Materials; Mesoscale modelling; Three-dimensional modelling.

\section{Introduction}

The development of materials for advanced engineering applications requires full understanding of the material response to different operational and environmental

*e-mail: ivano.benedetti@unipa.it 
conditions and, in many instances, the knowledge of their failure mechanisms is of crucial importance. Component level phenomenological models may not always be able to predict complex materials behaviors, especially if damage initiation and evolution are of concern. It is today widely recognized that these aspects may be better understood if the features of the material microstructure are considered and brought into the modelling framework. The link between microstructure and material macroscopic properties, the structure-property relationship ${ }^{1,2,3,4}$, is technologically interesting as it may provide valuable information for the design of enhanced materials ${ }^{5}$.

Polycrystalline materials (metals, alloys or ceramics) are commonly used in engineering applications. Their microstructure, at the grain scale, is characterized by the grain morphology, size distribution, anisotropy and crystallographic orientation, by the presence of flaws and porosity and by physical and chemical properties of the intergranular interfaces ${ }^{6}$, which also have a direct effect on the initiation and evolution of damage.

The behavior of polycrystalline materials at the microscale can be studied using experimental $7,8,9,10,11,12,13,14,15$ and computational techniques ${ }^{4,16,17}$. Much research has been carried out for developing numerical models for the analysis of polycrystalline microstructures and their failure processes. Until recently, the development of truly three-dimensional (3D) models was hindered by excessive computational requirements. In recent years, however, computational micromechanics has experienced a remarkable acceleration, due to the wider affordability of high performance parallel computing, thus favoring the advancement of the subject ${ }^{18,19,20,21}$.

There are several scientific and technological reasons for the interest in truly 3D polycrystalline microstructural models ${ }^{22,23,24,25}$ :

- 3D models are required for understanding inherently 3D complex microstructural phenomena: the influence of the geometry on the microcracking evolution; the competition between different failure modes, e.g. interand transgranular brittle propagation or the ductile-to-brittle transition; the grain-to-grain propagation of cleavage fracture ${ }^{26}$; the plastic deformation of the individual crystals.

- In some studies, simplified 3D geometries were initially generated extruding 2D grain topologies, sometimes obtained from the experimental observation of micro-specimen surfaces. Although this may be suitable for very thin specimens or films, it has been demonstrated that specimens built from an identical surface grain structure, but with different internal morphology, give raise to large discrepancies between the various solutions ${ }^{27,28}$, which justifies the trend towards fully 3D models;

- Computational investigations complement experimental techniques that, in the $3 \mathrm{D}$ case, may be particularly complex and expensive, especially when damage and failure are considered.

- An increasing number of applications, in various fields, require the man- 
ufacturing of micro-components whose overall dimensions are comparable with the grain size, thus requiring accurate analysis at the grain scale level.

In this review, a brief survey of recent works on grain scale 3D mechanical modelling of polycrystalline materials is presented. Other reviews on the micromechanics of polycrystalline materials exist: Cailletaud et al. ${ }^{29}$ provided some elements of micromechanics; Crocker et al. ${ }^{22}$ gave a survey of computational modelling of fracture in polycrystalline materials; a very comprehensive review of crystal plasticity was provided by Roters et al. ${ }^{17}$; Simonovski and Cizelj ${ }^{30}$ shortly reviewed grain scale approaches to polycrystalline materials. The present review is mainly focused on $3 \mathrm{D}$ modelling and is intended to provide the reader with a quick introduction this rapidly evolving field.

The paper is organized as follow. An overview of the main techniques used to generate artificial polycrystalline microstructures is given in Section 2, where simple regular microstructures, random Voronoi tessellations, structures generated through grain-growth algorithms and experimentally reconstructed microstructures are discussed. Section 3 gives an overview of the modelling strategies used for modelling the polycrystalline aggregate, from the simplistic linear elastic models to the more sophisticated models including crystal plasticity and damage. Some works on multiscale modelling involving polycrystalline materials are briefly discussed in Section 4. Some general discussion is given in Section 5, before the Conclusions.

\section{Generation of model microstructures}

The creation of a computer model of the polycrystalline microstructure requires at least two steps:

- the generation of the microstructural morphology, described by sets of points, grain edges, faces and volumes, which define the microstructural topology;

- the generation of an affordable mesh of the morphology.

If the analysis of large deformation is of interest, an additional step may be the remeshing, addressed at reconstructing a high-quality mesh from a deformed mesh comprising poor-quality elements ${ }^{31}$.

The problem of the generation of a suitable virtual microstructure, morphology and mesh, is particularly relevant, especially when the analysis of a relevant number of grains in the three-dimensional case is of interest. Analysis of this kind are of interest, for example, for predicting the occurrence of rare events in the polycrystalline microstructure ${ }^{32}$. Since the analysis of $3 \mathrm{D}$ micro-structures still remains a computationally challenging task, the quality of the generated microstructure and the ability to deal with contingent artifacts, for example the presence of small edges/faces that may challenge the round-off limit of the machine, due to the induction of undesired overly mesh refinement, are so relevant to affect the feasibility and success of the subsequent analysis strategy. 
In the present section, the generation, meshing and re-meshing of virtual 3D microstructures are discussed. Different methods for the generation of a virtual morphology are discussed, in an order based on the ability to represent accurately the features of the microstructure: at one hand, there are simplistic highly idealized representations, in which the grains are represented as regular morphologies (cubes, octahedra, etc.); at the other hand, there are highly sophisticated experimental reconstructions, with sub-micrometer resolution, able to represent also microstructural defects. It is worth highlighting that a given representation may be more or less accurate depending also on the specific task of the analysis: while a simplistic morphology may produce accurate results in a homogenization framework, where material macro-properties are inferred from the meso-scale, the analysis of microdamaging and micro-cracking processes is more likely to require a more accurate representation of the micro-features, for example grain boundaries and defects.

\subsection{Generation of virtual morphologies}

A virtual representation of the polycrystalline morphology can be generated resorting to: a) simplistic regular morphologies (cubes, dodecahedra, truncated octahedra); $b$ ) analytically generated random morphologies, notably Voronoi tessellations; $c$ ) computational simulation of grain growth; $d$ ) experimental techniques for material characterization.

\subsubsection{Regular simplified morphologies}

Simplified grain morphologies have often been used in the literature, especially in the context of homogenization theories ${ }^{33,34}$, where the main objective of the analysis is the establishment of a link between the material micro- and macro-properties, i.e. the structure-property relationship, or for texture evolution predictions ${ }^{35,36}$, in crystal plasticity finite element analyses. Some example regular microstructures are shown in Fig.1. It is worth mentioning that some materials naturally present a regular micromorphology; in these cases the regular morphology is not a simplified representation, but the accurate microstructure reproduction.

In Kalidindi et al. ${ }^{37}$, simple 2D square elements were used to represent the grains in a finite element crystal plasticity computation, but the elements incorporated complete 3D slip systems. Beaudoin et al. ${ }^{38}$ developed a hybrid FE formulation for polycrystal plasticity of FCC metals, to investigate the link between micro and macro variables of deformation and stress. In their model, a single brick finite element is used to represent either a whole (averaged) polycrystal in a continuum mechanics framework or, more interestingly for the present study, a single grain of a polycrystal, including effects related to the crystallographic anisotropy. Interelement continuity was exactly enforced on the aggregate, while inter-element equilibrium of tractions was satisfied in an averaged sense, via weighted residuals, which 
explains the hybrid appellation of the formulation. Besides other applications, the model was used to investigate the channel die compression of an aggregate with $16 \times 16 \times 16$ brick elements, each representing a grain with its own crystallographic orientation. The aggregate texture evolution was investigated and satisfying results were reported and compared with other models predictions (Taylor and relaxed constraints models) and some experimental data. The work highlighted the suitability of the formulation for cases where significant spatial variations exist in the constitutive response within the investigated domain, as it is the case of polycrystals.

The model was then employed by Sarma and Dawson ${ }^{39}$, in a series of simulations addressed at studying the effect of the interactions between a crystal and its neighbors on its own deformation and the overall texture evolution. Although the grains specific morphology was not considered, the results showed that the interactions among crystals play an important role in determining how the applied macro-deformation is redistributed among the grains. These observations were then used ${ }^{40}$ to incorporate the effects of interactions of each grain with its neighbor grains, computing its compliance relative to a set of neighboring crystals, in a crystal visco-plasticity context. The model was used to successfully predict the texture evolution under conditions of plane strain compression. It is worth noting here that that in the concluding remarks the importance of incorporating crystal shape effects into the model was highlighted by the authors.

Polycrystalline aggregates are idealized as simple three-dimensional arrangements of grains in ${ }^{41}$, where many elements per grain are used to represent non-uniform deformations within individual grains, seen as domains separated by boundaries of high misorientation. A crystal plasticity FE formulation is used to examine localized deformation in fcc polycrystals.

Similar models, i.e. FE based averaging procedures developed initially as an improvement over classical averaging schemes (e.g the Taylor model) for modeling the constitutive behavior of polycrystal aggregates with a known distribution of lattice orientations and a known single crystal constitutive behavior, were tested by Bachu and Kalidindi ${ }^{42}$, who used the three-dimensional FE model developed in ${ }^{43}$ to provide a direct quantitative comparison of the finite element $(\mathrm{FE})$ predicted textures

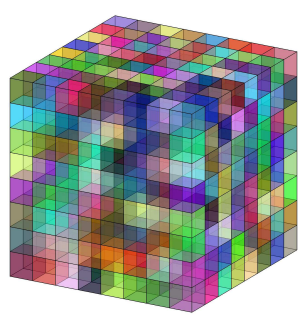

a

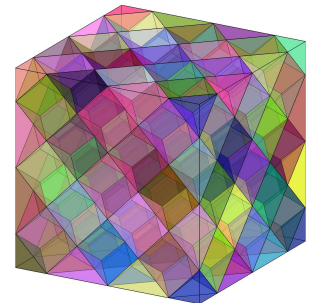

b

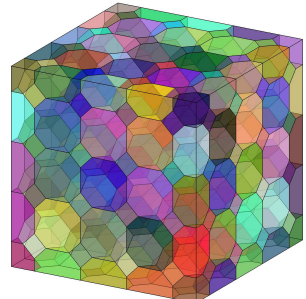

C

Fig. 1. Example regular morphologies: a) cubic grains; b) rhombic grains; c) truncated octahedra. 
with some experimental results. Analogously, Van Houtte et al. ${ }^{44}$ used, in a 3D framework, the $\mathrm{FE}$ model developed $\mathrm{in}^{37}$ to assess an alternative grain interaction model (Lamel model). Also in these cases, the shape of the grains was not accurately represented and each grain was modelled with one or more finite elements of regular (usually cubical) shape and specific crystallographic orientation, although the need for more accurate representation of the micro morphology was advocated by Van Houtte et al. ${ }^{44}$.

A more sophisticated, although still idealized, representation of the grains was assumed by Mika and Dawson ${ }^{45,46}$, who represented fcc single crystals as rhombic dodecahedra, i.e. 12-sided, space-filling polyhedra. Each crystal was then discretized withe tetrahedral finite elements, the material constitutive behavior was rate dependent crystal slip and compatibility and equilibrium were enforced in a weak sense using hybrid finite element methodology. One of the declared objectives of the investigation was the analysis of the influence of the crystal assumed shape on the interactions between a crystal and its neighborhood, and the comparison with previous analyses conducted adopting brick shaped crystals. Moreover, these studies highlight in their conclusions that finer discretizations, and arguably morphologies, are better equipped for a detailed investigation of local neighborhood effects.

Three-dimensional brick elements and tetrahedral elements arranged to form space filling rhombic dodecahedra were employed $\mathrm{in}^{47}$, addressed to the assessment of the texture development predictions of Taylor and finite element models in BCC iron. A regular microstructure comprised of truncated octahedra, also referred to as a tetrakaidecahedra or mecons, each representing a single grain was employed by Jivkov et al. ${ }^{48}$ to study intergranular stress corrosion cracking in stainless steel with finite elements. The choice of the truncated octahedron as representative of a single grain is motivated by the authors by the fact that, among all the 3D space filling polyhedra, this polyhedron presents a coordination number (number of neighbor grains) closest to those observed in reality. With this assumption, a polycrystalline aggregate is represented by an assembly of identical cells. It was highlighted that the suggested representation of the microstructure reduces substantially the computational requirements for the simulation of the crack propagation in large grain aggregates. Extension to more general (not regular) microstructures was also suggested.

Grains shaped either as cubes or truncated octahedrons, and different levels of mesh refinement are used in ${ }^{49}$, where the polycrystal is represented by a finite element mesh loaded by periodic boundary conditions. Simulations rely on a simplified implementation of crystal plasticity and it was concluded that truncated octahedral grains yield improved predictions compared to those with cuboidal grains.

Ritz and Dawson ${ }^{34}$ considered grains of cubic, rhombic dodecahedral and truncated octahedral shape and used an elasto-visco-plastic finite element model to compare the mechanical response of meshes formed with these grain shapes. They examined the influence of the grain shape on the aggregate response, in terms of local stress 
variations and aggregate elastic moduli, and concluded that elastic anisotropy in the bulk material was not dependent on grain shape, while consistent trends were observed in intragranular and intergranular stress distributions across these mesh definitions.

\subsubsection{Voronoi tessellations}

Voronoi tessellations have been extensively used, both in two and three dimensions, to provide first order approximations of random polycrystalline microstructures. It has been shown that the Voronoi tesselations, built on a set of points randomly scattered within a given volume, possess statistical features that make them topologically close to real ceramic and metallic microstructures, as reported for example by Kumar et al. ${ }^{50,51}$, who used 3D Voronoi tessellations to evaluate the effective thermal expansion coefficient and the micro-stress distribution within a polycrystalline aggregate.

Among randomly generated morphologies, Voronoi tessellations present the advantages of being analytically defined and having straight triple lines and flat grain faces, which appears quite appealing for numerical/computational purposes. Given a bounded domain $\mathscr{C} \subset \mathbb{R}^{3}$, and a set of generator points $P=\left\{\boldsymbol{x}_{k} \in \mathscr{C}: k=1, \ldots, n\right\}$, a Voronoi tessellation of $\mathscr{C}$ is given by the union of the polyhedra $\mathscr{G}_{k}$ defined by the condition

$$
\mathscr{G}_{k}=\left\{\boldsymbol{y} \in \mathbb{R}^{3}:\left\|\boldsymbol{y}-\boldsymbol{x}_{k}\right\|<\left\|\boldsymbol{y}-\boldsymbol{x}_{j}\right\| \forall j \neq k\right\}
$$

The Voronoi cells are convex polyhedra bounded by flat polygonal faces. Two adjacent Voronoi cells share a tessellation face, three cells share an edge and four cells meet at a point called vertex, Fig.2. Voronoi tessellations have an interesting physical interpretation: they can be seen as structures generated by a metallurgical solidification process in which all the grains nucleate at the point seeds at the same time, the seeds maintain the same position in space during the process, the solidification rate is uniform and isotropic for all the grains and stops when two grain boundaries meet. The topology and morphology of the tessellation depend on the arrangement of the set of point seeds scattered in the container $\mathscr{C}$. Regular morphologies (for example cubes, octahedra, dodecahedra) can be obtained arranging the seeds are arranged in a regular grid. However, in the case of polycrystalline microstructures, a set of randomly distributed points is generally used to generate the tessellation, which is then referred to as Poisson-Voronoi tessellation.

Several examples of use of Voronoi tessellations for polycrystalline materials are present in the literature. Kozaczek et al. ${ }^{52}$ used the Poisson-Voronoi tessellation to study the effect of the microstructural topology on the grain-boundary stress distribution in Alloy 600 polycrystalline materials. They generated two sample pure microstructures (without defects or precipitates), with 250 and 500 grains respectively, and analyzed the local concentrations of stresses at the vertices of $3 \mathrm{D}$ grains 
as a function of the misorientation angle between the grains themselves. Barbe et al. ${ }^{53,54}$ used $3 D$ Voronoi polyhedra for the analysis of intergranular and intragranular fields and also in recent works including modelling of phase transformations at the microscal ${ }^{55}$. Three-dimensional Voronoi algorithms have been used by Nygårds and Gudmundson ${ }^{56}$, to generate a periodic polycrystalline microstructures for FE simulations of a two-phase steel, by Nygårds ${ }^{57}$, who generated periodic models for investigating the number of grains necessary to homogenize elastic polycrystals with cubic symmetry and by Kanit et al. ${ }^{58}$, who proposed a quantitative definition of the RVE size carrying out FE simulations on two phase Voronoi mosaics for linear elasticity and thermal conductivity problems. Zhao and Tyron ${ }^{59}$ used a Voronoi algorithm to perform a statistical analysis of microstress distribution with single- and multi-phase polycrystalline metallic aggregates. 3D Voronoi polyhedra have been employed by Diard ${ }^{60}$ for studying the plasticity of polycrystalline aggregates through crystal plasticity finite elements and by Zhang et al. ${ }^{61}$, who simulated microplasticity-induced deformations in uniaxially strained ceramics. Threedimensional Voronoi tessellations have also been used by Kamaya et al. ${ }^{62}$, who performed a statistical analysis of grain-boundary stresses in a microstructure comprised of 100 grains, by ${ }^{63,64,65,32}$, who modeled bainitic steel with crystal plasticity finite elements, by Luther and Könke ${ }^{66}$, who developed an algorithm to generate polycrystals with arbitrary grain size distribution functions for studying brittle intergranular damage in metallic polycrystals, by Musienko and Cailletaud ${ }^{67}$, to study intergranular stress corrosion cracking, transgranular cracking in a crystal plasticity framework, by Weinzapfel et al. ${ }^{68}$, who presented a finite element model for analyzing subsurface stresses in an elastic half-space subjected to a general Hertzian contact load, with explicit consideration of the material microstructure topology, by Bomidi et al. ${ }^{21}$, who developed a 3D finite element model to investigate intergranular fatigue damage of micro-electromechanical systems (MEMS) devices and to account for the effects of topological randomness of material microstructure on fatigue lives and by Benedetti and Aliabadi ${ }^{69,70}$, who studied the evolution of intergranular damage and cracking through cohesive-frictional boundary elements.

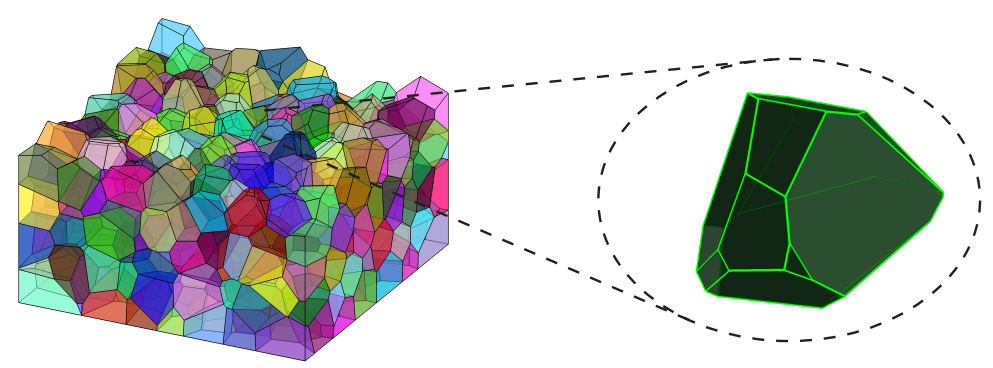

Fig. 2. An example of micromorphology generated through Voronoi tessellation. The individual grains are convex polyhedra with flat polygonal faces. 
It is worth noting that Voronoi tessellations are also frequently taken as initial microstructures in many thermodynamics-based models (phase field, level-set, ...) meant at describing recrystallisation or phase transformation in polycrystals $71,72,73,74$.

Some different aspects have been investigated and should be considered when generating Voronoi tessellations with the aim of modelling polycrystalline microstructures:

- the initial set of seed points must be scattered, or packed, so to eventually generate tessellations with a suitable statistical grain size distribution;

- the topology of the tessellation should be as clean as possible from excessively small entities, either edges or faces, which, although mathematically consistent, can become problematic from a computational point of view, challenging the machine round-off precision or inducing overly mesh refinement.

Several studies have been performed on various topological and morphological statistical features of three-dimensional Voronoi aggregates, to assess their ability to represent some spatial structures in various fields of investigation $75,76,77,78$. In the specific case of polycrystalline microstructures, on the basis of some experimental investigations, some authors ${ }^{7}$ have highlighted that Voronoi tessellations underestimate the grain size distribution and overestimate the number of faces per grain. Some of these issues can be corrected through the application of correction schemes $^{31}$ that slightly modify the topology of the aggregate, and then the statistical distributions of some features, at the cost of some pre-processing complication. It is also possible, to some extent, to modify the topological and morphological features of random aggregates controlling the initial distribution of generating points ${ }^{79}$, introducing a hardcore constraint to avoid points too close to each other and then distorted grains. In any case, the Voronoi tessellations retain the advantages of simplicity and suitability for numerical treatment. Algorithms for packing particles together to provide tessellations with more realistic grain size distributions have been proposed also by Fan et al. ${ }^{80}$, who suggested Voronoi diagrams in the Laguerre geometry based on random closed packing of spheres, with volumes following a log-normal distribution, as a better approximation of real materials with respect to Poisson-Voronoi diagrams, by Nouri et al. ${ }^{81}$, who developed two different algorithms based on molecular dynamics and on the discrete element method and by Lavergne et al. ${ }^{82}$, who combined dense-sphere packing with Power diagrams.

Three-dimensional Voronoi tessellation can be generated using different software packages available in the open domain. Examples are provided by Neper ${ }^{31}$ (http://neper.sourceforge.net/), Voro++ 83 (http://math.lbl.gov/voro++/), Qhull ${ }^{84}$ (http://www.qhull.org/). Some packages, for example Neper, also provide meshing procedures for FE analysis. In the choice of a package, it is important to consider whether the way the tessellation is generated and stored is suitable for the particular considered application. Some 
packages, e.g. Voro++, generate the tessellation as a set of individual grains, with their own edges and faces and list of neighboring grains, while others, e.g. Qhull, represent the tessellation as a collection of vertices connected by edges, so that the identifications of individual grains may result more involved. The preference between one representation or the other depends then on the target application.

\subsubsection{Computational simulation of grain-growth}

Voronoi tessellation are known to provide a first order approximation of polycrystalline microstructures. Nevertheless, they still generate highly idealized grain morphologies and this is apparent considering that each Voronoi cell is a convex polyhedron bounded by flat convex polygonal faces. A better approximation of the real grain morphology, in a given material, can be obtained by directly simulating the grain growth process, on the basis of boundary energy minimization principles. A recent review of grain-growth simulation techniques, with a discussion of advantages and drawbacks of each method, has been given by Miodownik ${ }^{85}$.

In recent years, several techniques for the computer simulation of grain growth have been developed, including Monte Carlo Potts models, vertex tracking, front tracking, cellular automata and phase-field approaches. These methods were initially developed for the $2 \mathrm{D}$ case. However, the affordability of higher computational power has recently promoted the extension to the non-trivial 3D case. Three-dimensional aggregates with a relevant number of grains have been successfully addressed using Monte Carlo Potts models $86,87,88,89,90$. A Monte-Carlo grain growth algorithm was used by Sarma et al. ${ }^{91}$, who generated an initial microstructure for FE simulations of cold deformation at the mesoscale in a crystal plasticity framework. Also other techniques such as boundary-tracking approaches ${ }^{92,93,94}$, vertex tracking ${ }^{95,96,97}$ and cellular automata ${ }^{98}$ have been successfully developed.

A versatile and mature method for grain-growth simulation, along with Monte Carlo Potts models, is the phase-field technique, particularly adequate in the presence of multiple phases or gradients of concentration, stress or temperature. A 3D phasefield model has been developed by Krill III and $\mathrm{Chen}^{99}$. Suwa et al. ${ }^{100}$ developed a 3D phase-field model of grain growth for materials containing finely dispersed second-phase particles. They also investigated the effect of anisotropy in grainboundary mobility on growth kinetics and morphology of grain structure ${ }^{101}$ and performed 3D simulations of normal grain growth by using the multi-phase-field (MPF) model, accelerated by parallel coding techniques ${ }^{102}$. For reducing the computational requirements of phase-field models, Vanherpe et al. ${ }^{103}$ proposed a sparse bounding box algorithm and applied it to a 3D model for grain growth in presence of second-phase particles. An introduction to phase-field modelling of microstructural evolution is given in Moelans et al. ${ }^{104}$, while a recent review of the technique is given by Loginova and Singer ${ }^{105}$. 


\subsubsection{Experimental characterization of three-dimensional microstructures}

For the generation of virtual polycrystalline microstructures, at the other end of the picture with respect to highly idealised and simplistic representations, in terms of accuracy of the generated morphology, there are three-dimensional reconstructions based on more or less sophisticated experimental techniques. The experimental characterization has traditionally been based on optical microscopy, scanning electron microscopy (SEM) or transmission electron microscopy (TEM) of two-dimensional sections of three-dimensional micro-specimens. Any reconstruction technique must be able to provide information on both the micro-morphology of the aggregate and its crystallographic features (crystallographic orientation, presence of different phases, etc.). A truly three-dimensional reconstruction is still a challenging task, but some interesting advancements have recently taken place.

The techniques for three-dimensional microstructural reconstruction can be classified into destructive and non-destructive techniques. Non-destructive reconstructions are particularly relevant when the study of polycrystalline deformation, texture development or micro-damage and micro-cracking evolution is of interest: it is apparent how any destructive technique interferes, for example, with the propagation of an intergranular micro-crack, making as a matter of fact impossible the study of its evolution. On the other hand, destructive reconstructions of large polycrystalline aggregates allow the extraction of statistical data that can be used to assess and improve the generation of artificial microstructure.

Among destructive techniques, an important role for three-dimensional characterization of materials, is played by serial sectioning: the relevant morphological and crystallographic information is collected, by means of various acquisition techniques, from a sequence of stacked two-dimensional sections of the material specimen and then post-processed to generate an as much as possible accurate microstructural representation ${ }^{8,106}$. Strictly speaking, serial sectioning refers to the material removal operations that allow to access sequential layers of the micro-specimen. The micro-polishing can be performed using different techniques (mechanical, chemical, laser-based, etc.) and care must be taken to avoid altering or destroying the microstructural features that must be measured. After a specific layer is revealed, the $2 \mathrm{D}$ trace of the individual grains on the considered plane is acquired through optical or electronic microscopy, while the information about the crystallographic orientation is provided by means of Orientation Imaging Microscopy (OIM), such as Electron Back Scattering Diffraction (EBSD). If a 3D characterization is pursued, the information collected from the different accessed layer must be combined to provide a consistent representation of the microstructure, whose quality depends on different factors such as the the accuracy and automation of the polishing techniques, the sampling resolutions, both in-section and in-depth, the algorithms used for post-processing the collected data and avoiding possible artifacts, such as grain overlapping. Automated serial sectioning is a flexible, low-cost technique for rapidly obtaining three-dimensional (3-D) microstructural data in a wide variety of mate- 
rials, as described for example by Spowart et al. ${ }^{107,108}$. Digital imaging processing and segmentation techniques can be used to address reconstruction issues ${ }^{109}$. Serial sectioning has been consistently used in the recent literature for studying polycrystalline microstructures. Lewis et al. ${ }^{110}$ used a combination of serial sectioning with EBSD for the reconstruction of individual grains in a commercial superaustenitic stainless steel. Second-phase $\sigma$ particles features were also analyzed. The obtained results were intended to provide boundary conditions for atomistic calculations of specific grain boundary structures as well as the basis for mesoscale image-based models of mechanical behavior of the microstructures. Rowenhorst et al. ${ }^{111}$ combined EBSD analysis with serial sectioning to allow for direct, quantitative investigations into the 3D morphology and 3D crystallography of coarse martensite crystals formed in HSLA-100 steel. An integrated framework for the automated analysis of polycrystalline microstructures based on serial sectioning has been developed by Groeber et al. ${ }^{9,112,11,113}$, based on a dual-beam focused ion beam-scanning electron microscope (DB FIB-SEM) equipped with an EBSD system. A similar approach has been developed and used by Zaefferer et al. ${ }^{114}$. Similar techniques are being increasingly used also in geology for the characterization of geological, also polycrystalline, materials ${ }^{115}$. An introduction to the use of the use of focused ion beam microscopy in materials science can be found in Munroe ${ }^{116}$.

The output of serial sectioning has often been used to generate computational models for finite element (FE) analysis. Sumigawa et al. ${ }^{117}$ used repeated polishing and OIM to obtain the crystalline orientation and the three-dimensional shape of each grain of an aggregate, to perform a FE analysis and study the characteristic slip behavior near the grain boundary of a copper polycrystalline specimen subjected to high-cycle fatigue. In Lewis and Geltmacher ${ }^{118}$ a 3D spatial and crystallographic reconstruction was generated using optical microscopy, serial sectioning, and EBSD for an austenitic steel microstructure. The generated output was incorporated into an image-based FE model to simulate the mesoscale mechanical response of the real microstructure and specific 3D visualization techniques were used to identify critical microstructural features at which the onset of plasticity, failure and fracture are predicted to occur. Bhandari et al. ${ }^{119}$ developed a methodology in which realistic 3D polycrystalline microstructures, generated through the DB FIB-SEM serial sectioning technique developed by Groeber et al. ${ }^{9,112}$, are discretized into consistent 3D tetrahedral finite elements by a mesh generator provided with resolution control, for ensuring meaningful computational analysis in microstructureproperty evaluation. The reconstruction uses primitives in CAD modeling based on hierarchical geometrical representation and requires data cleanup, interface point identification, parametric polynomial and NURBS function based surface patch reconstruction, generalized cell-decomposition, geometric defeaturing and gap-overlap removal. Musienko et al. ${ }^{120}$ used a technique based on micro-polishing, SEM and Orientation Image Microscopy (OIM) for performing a crystal plasticity FE analysis of a three-dimensional copper specimen. The three-dimensional microstructure of 
a bcc $\beta$-Ti alloy was generated combining serial sectioning, high-resolution optical microscopy, EBSD and image processing algorithms by Qidwai et al. ${ }^{121}$, who also provided a short overview of image-based computational modelling for polycrystals. The reconstructed microstructure was incorporated into large-scale crystal plasticity FE models, to study microstructureyield correlation in the considered titanium alloy. Serial sectioning and SEM-ESBD measurements were used by Nakamachi et al. ${ }^{122}$, to experimentally characterize the 3D microstructure of a $99 \%$ pure iron sheet metal and generate an RVE to be used in a homogenization based multiscale finite element analysis of sheet metals.

It is worth noting that in some cases, OIM alone is sufficient to reconstruct the morphology of the specimen, without resorting to polishing operations. However, this is the case of simple columnar or quasi-columnar specimens, such as those considered for example by Bhattacharyya et al. ${ }^{123}$, who used Back-Scattered Kikuchi Diffraction patterns and SEM to obtain orientation imaging micrographs of polycrystalline aluminum samples to be used in a crystal plasticity study. You et al. ${ }^{124}$ performed a detailed comparative experimental/computational analysis of the tensile deformation of a 316LVM stent strut-like specimen. Due to the typically small cross section of a stent strut, it was possible to develop full 3D models of the specimen without resorting to any polishing and using only SEM, strain mapping and OIM.

In other instances, orientation imaging micrographs of selected external specimen surfaces have been used, in conjunction with space filling algorithms and Voronoi tessellations, to generate statistical reconstructions of the internal 3D microstructure, as suggested by Brahme et al. ${ }^{125}$. Using the same idea, St-Pierre et al. ${ }^{126}$ and Gérard et al. ${ }^{127}$ presented a methodology to create realistic 3D microstructures of polycrystals: based on 2D EBSD analysis, the parameters necessary to describe a microstructure were identified as grain size, grain shape and crystallographic orientation distributions. Then, a methodology was developed to extend this surface observation to a realistic volumetric description, necessary to simulate 3D microstructures. The generated microstructures were tested and optimized through FE simulations. The approach has been validated by comparing multiple slices of a virtual 3D microstructure with an experimental surface observation.

Non-destructive techniques for the 3D reconstruction of polycrystalline microstructures include $X$-ray tomography ${ }^{128,129,130,131}, X$-ray diffraction microscopy ${ }^{132}$, differential aperture X-ray microscopy ${ }^{133}$.

X-ray tomography has been used to extract information on the 3D micro-topology and micro-morphology of polycrystalline materials and it can provide 3D density and chemical distributions with sub-micrometre resolution. This data is useful for the visualization of the microstructure, but it must be complemented with additional crystallographic information, when the study of the structure-property link or other mechanical aspects are of interest. Ludwig et al. ${ }^{134}$, for example, used synchrotron radiation X-ray micro-tomography, complemented with EBSD measurements of the grain orientation on the sample surface, for the 3D visualization and analysis of the interaction of a short fatigue crack with the grains of a cast $\mathrm{Al}$ alloy. 
The visualization of the grains was obtained by decorating the grain boundaries by liquid Ga, used as a selective contrast agent. The complex 3D shape of the crack were correlated with the grains structure. Dobric et al. ${ }^{7}$ used absorption-contrast X-ray micro-tomography, to perform large-scale microstructural characterization of polycrystalline aluminum. The aluminum sample was doped with $S n$, with the aim of enhancing the grain boundary X-ray contrast, due to its segregation at the grain boundaries. Suitable refinement schemes, based on grain-growth algorithms, were employed to extract reliable information about the topology, morphology, grain size of nearly 5000 individual grains in polycrystalline $A l-S n$. It is interesting to note that, on the basis of their work, the authors concluded that the distributions and averages of available data deviate significantly from the corresponding microstructural parameters of 3D Poisson-Voronoi tessellations, often used to model polycrystalline samples, and that much better agreement was observed with microstructures generated by the computer simulation of three-dimensional grain growth.

Larson et al. ${ }^{133}$ developed a 3D X-ray technique with polychromatic synchrotron X-ray micro-beams, called differential aperture X-ray microscopy (DAXM), for the characterization of single-crystal, polycrystalline, composite and functionally graded materials. Using the technique, they were able to probe local crystal structure, orientation and strain tensors with submicrometre spatial resolution and demonstrated the methodology on polycrystalline aluminium and cylindrically bent silicon. As the authors concluded, the availability these high-resolution measurements provided a direct, and previously missing, link between the actual material microstructure and evolution and the results of numerical simulations and multi-scale modelling of microstructure and evolution on mesoscopic length scales. This is possible because this reconstruction technique provides non-destructively morphological, crystallographic and deformation information, allowing then to follow the evolution of the microstructure under external actions: it is worth noting that this kind of analysis/assessment is not possible with destructive techniques, which only allow pre- or post-deformation measures.

A range of techniques for 3D non-destructive material characterization have been developed at the Ris $\varnothing$ National Laboratory, Denmark ${ }^{135}$. The methods are based on the diffraction of high-energy X-rays and have been implemented, in collaboration with the European Synchrotron Radiation Facility (ESRF), in a methodology known as three-dimensional X-ray diffraction (3DXRD) microscopy ${ }^{136}$.

A technique closely related to 3DXRD, called diffraction contrast tomography (DCT), has been developed by King et al. ${ }^{10,137}$ in collaboration with the Ris $\varnothing$ group ${ }^{138,139,12}$, and it combines the principles of X-ray diffraction imaging, threedimensional X-ray diffraction microscopy (3DXRD) and image reconstruction from projections. DCT provides access to 3D grain shape and crystallographic orientation and it can be applied to a large range of polycrystalline materials, provided some conditions on grain size and texture are fulfilled. The straightforward combination with high-resolution micro-tomography opens interesting new possibilities for the observation of microstructure related damage and deformation mechanisms in these 
materials, as it has been shown by King et al. ${ }^{10}$, who studied the interaction between inter-granular stress corrosion cracking and microstructure. Model microstructures generated by DCT have also been used by Simonovski and Cizelj ${ }^{18,19,20,30}$ to study intergranular stress corrosion cracking.

\subsection{Microstructure meshing and remeshing}

Once the microstructural morphology has been generated, the construction of a computational mesh of adequate quality constitutes a non trivial task. The challenge is the generation of a mesh able to represent, with sufficient accuracy, the local fields, but with a reasonable number of degrees of freedom. Except the simple case of regular microstructures, whose regularity simplifies the meshing process, the general case of random or experimentally reconstructed microstructures requires special attention, due to the recurrence of entities (grain edges or faces) which may vary considerably with respect to their average size, thus challenging the homogeneity of the mesh itself, or due to the presence of experimental artifacts, such as gaps or overlaps, that need to be removed before generating the mesh itself.

Focusing on the case of random Voronoi tessellations, either structured or unstructured meshes have been used in the literature ${ }^{140}$. Structured meshes, employed in many works ${ }^{53,54,57,60}$, use elements of regular shape, e.g. cubes, to mesh the entire region of interest and attribute to each element the crystallographic properties of the grain to which they belong, based on the previously generated tessellation. It is obvious that this approach cannot represent with sufficient accuracy the interface between two grains and also with very refined meshes the introduction of intergranular artifacts is unavoidable. Unstructured meshes ${ }^{50,51,79,59}$, on the other hand, are built starting from the grains morphology. Usually, the grain edges (1D) are meshed first, to provide the seeds for the meshing of the grain faces (2D), which in turn provide a constraint for the interior domain (3D). Their advantage is that, being built on the morphology itself, are able to represent without artifacts the intergranular interfaces.

As mentioned above, random tessellations show remarkable variabilities in grain size and shape, which are representative of real morphologies. This aspect makes them particularly challenging to mesh with good-quality elements. As a consequence, as pointed out by Quey et al. ${ }^{31}$, free meshing of Voronoi tessellations has been applied almost exclusively to relatively small polycrystals $\left(<10^{3}\right.$ grains $)$ and used primarily in the context of small deformation studies ${ }^{60,67,79}(<1 \%)$. In this context, mesh homogeneity has been usually pursued linking the mesh to the average edge length $\bar{L}_{e}$, which is a feature of the tessellation, by introducing a discretization parameter $d_{m}$, so that the generic tessellation edge of length $L_{e}$ is split in a number of segments given by

$$
n_{s}=\text { round }\left(d_{m} \frac{L_{e}}{\bar{L}_{e}}\right)
$$


where $\operatorname{round}(x)$ is the function returning the value of the argument rounded to the integer closer to $x$ itself (excluding zero). The parameter $d_{m}$ then defines the number of segments in which the average length cell edge is split, and it is then a measure of the mesh density. After edge splitting has been accomplished, the segment vertices are used as seeds for the face triangulation and the face mesh is used as constraint for the volume discretization. This procedure has been used for example by Fritzen et al. ${ }^{79}$, who developed a numerical methodology for the generation of 3D periodic meshes with variable mesh density, for the homogenization of polycrystalline aggregates. Benedetti et al. ${ }^{69,141,70}$ used this strategy, together with other improvements addressed at ensuring the generation of good surface triangulations, to provide a mesh of the surface only of the grains, to be used in the boundary integral formulation of the polycrystalline problem.

Although this simple technique may provide acceptably regular meshes in the case of small aggregates (few grains), the case of large aggregates or large deformations requires further attention. The unavoidable presence of small entities, for example short edges, is generally responsible for the induction of overly mesh refinements, with obvious disadvantages in terms of mesh affordability. This issue has been addressed by Quey et al..$^{31}$, who presented an original methodology for the generation, meshing and remeshing of large-scale three-dimensional random polycrystals. They generated the microstructure through Voronoi tessellation and proposed original approaches for: $a$ ) geometry regularization, by removing some geometrical morphological details; $b$ ) multi-meshing, by using simultaneously several meshing algorithms to optimize mesh quality; $c$ ) re-meshing, by which a new mesh is constructed over a deformed mesh and the state variables are transported, for large strain applications. The methodology was shown to generate meshes of optimal quality, with a reduced number of degrees of freedom. An alternative strategy, based on the use of level set functions for the generation of meshes with anisotropic refinement, was proposed by Resk et al. ${ }^{142}$, who also extended their technique to remeshing procedures in case of problems involving large deformations.

The meshing of experimentally reconstructed microstructures has been accomplished in several studies ${ }^{7,118,112,120,121,17}$ Bhandari and Ghosh et al. ${ }^{119,143}$ developed a sophisticated methodology for meshing 3D polycrystalline microstructures, generated with a DB FIB-SEM system. The grains are segmented as a collection of voxels using crystallographic orientation data and the surface topology is smoothed using polynomial and NURBS functions. A cell decomposition algorithm generates 3D grains, bounded by the regularized surface segments. Various strategies are used to remove artifacts, such as gaps or overlaps, and ensure grain compatibility. Finally, the reconstructed morphology is discretized into FE tetrahedral elements, and mesh-quality improvements are adopted to reduce the number of degrees of freedom. The methodology allows the control of the mesh resolution, which is an important aspect for micromechanical analysis.

Simonovski and Cizelj $^{18}$ developed a framework for automatic and parallelized gen- 
eration of FE models from discrete spatial data (voxels) obtained from experimental techniques, such as diffraction contrast tomography. The technique results particularly effective for complex polycrystalline aggregates, with explicit account for grain shapes and orientations. Special algorithms have been devised for processing voxel-based data, removing holes and ensuring consistency of the reconstructed morphology surfaces. Then, FE meshes of the reconstructed surfaces were generated, followed by interior volume meshing. The method assures conformal meshes between surfaces in contact and enables parallel (and the independent) meshing of the constituents.

\section{Computational modelling and analysis of polycrystalline microstructures}

The computational modelling and analysis of three-dimensional polycrystalline grain-scale structures has been tackled in several recent works. A variety of methods, differing for the complexity of the modelling strategy as well as for the scope of the investigation, have been proposed in the literature. Different models may differ for the constitutive laws used for the individual bulk grains: in some simplistic approaches, the grains are modelled as isotropic elastic domains, with different elastic constants in case of multiphase materials; other authors use anisotropic laws, while more advanced models include crystal plasticity, i.e. anisotropic plasticity taking place via shear on specific set of crystallographic planes. Also the modelling of interfaces varies from simple assumptions (perfect bonding between grains) to complex models such as cohesive laws embodying the possibility of damage initiation and evolution.

In this section, the following classification is proposed, for reviewing purposes: simple models, relying on the assumption of linear behavior of the constituent grains, and with no or simple modelling of the interfaces, are considered first; 3D models including crystal plasticity are reviewed after; more sophisticated works, including also the modelling of the inter-granular interfaces, and attention devoted to the analysis of damage or cracking, are considered last.

\subsection{Microstructures with linear elastic grains}

In several works the polycrystalline microstructure is modelled as an aggregate of linear isotropic grains, with different elastic modula, or as an aggregate of linearly orthotropic or anisotropic grains, with different orientation in space. In both cases, from the numerical point of view, irrespective of the strategy used to generate the micromorphology, the problem can be seen as a multi-domain or multi-region problem. The aggregate behavior is linear if the intergranular interface is modelled as a perfect bond between contiguous grains. However, more sophisticated aggregate behaviors emerge if the interface is modelled with traction-displacement relationships, allowing intergranular displacement jump. 
Without loss of generality, the constitutive equations for the grain material can be written

$$
\sigma_{i j}=c_{i j k l} \varepsilon_{k l}
$$

where the constants $c_{i j k l}$ denote the components of the stiffness tensor linking the stress tensor $\sigma_{i j}$ to the strain tensor $\varepsilon_{k l}$. If the compact Voigt notation is used to denote the elements of the elastic tensors, see for example ${ }^{144}$, the stiffness tensor can be written as a $(6 \times 6)$ matrix $\mathbf{C}=\left[C_{i j}\right]$, where $C_{11}=c_{1111}, C_{12}=c_{1122}$, $C_{16}=c_{1112}, C_{44}=c_{2323}$ etc. For the general orthotropic case, 9 constants are needed for the complete material characterization, being $C_{14}=C_{15}=C_{16}=0$, $C_{24}=C_{25}=C_{26}=0, C_{34}=C_{35}=C_{36}=0$ and $C_{45}=C_{46}=C_{56}=0$. The constitutive equations can then be written in the form

$$
\left[\begin{array}{l}
\sigma_{11} \\
\sigma_{22} \\
\sigma_{33} \\
\sigma_{23} \\
\sigma_{13} \\
\sigma_{12}
\end{array}\right]=\left[\begin{array}{cccccc}
C_{11} & C_{12} & C_{13} & 0 & 0 & 0 \\
C_{12} & C_{22} & C_{23} & 0 & 0 & 0 \\
C_{13} & C_{23} & C_{33} & 0 & 0 & 0 \\
0 & 0 & 0 & C_{44} & 0 & 0 \\
0 & 0 & 0 & 0 & C_{55} & 0 \\
0 & 0 & 0 & 0 & 0 & C_{66}
\end{array}\right]\left[\begin{array}{c}
\gamma_{11} \\
\gamma_{22} \\
\gamma_{33} \\
\gamma_{23} \\
\gamma_{13} \\
\gamma_{12}
\end{array}\right]
$$

where $\gamma_{i i}=\varepsilon_{i i}$ for $i=1, \ldots, 3$ and $\gamma_{i j}=2 \varepsilon_{i j}$ for $i \neq j$.

The main objectives of several studies assuming linear elastic single-crystal behavior were:

- the analysis of micro-stresses distributions within the microstructure, especially at the grain boundaries, likely to enable damage or fracture initiation at the mesoscale;

- the estimation of the macroscopic (effective, aggregate) material properties, emerging from the constituent properties, based on the previous determination of the representative volume element (RVE) size, often expressed in terms of number of grains necessary for the homogenization.

In a linear framework, the estimation of the effective material modula, is generally performed considering stress and strain volume averages ${ }^{3,16,145}$, defined by

$$
\left\langle\gamma_{i j}\right\rangle \doteq \frac{1}{V} \int_{V} \gamma_{i j}(\boldsymbol{x}) d V(\boldsymbol{x})=\frac{1}{2 V} \int_{\partial V}\left(u_{i} n_{j}+n_{i} u_{j}\right) d S
$$

and

$$
\left\langle\sigma_{i j}\right\rangle \doteq \frac{1}{V} \int_{V} \sigma_{i j}(\boldsymbol{x}) d V(\boldsymbol{x})=\frac{1}{V} \int_{\partial V} x_{i} t_{j} d S
$$

where the integrals over the RVE volume $V$ are transformed into surface integrals over the volume boundary $\partial V$ as consequence of the divergence theorem (the symbol $\doteq$ denotes a definition), if grains separation and body forces, respectively, are not present in the microstructure.

If macro-strains $\Gamma_{i j}$ and macro-stresses $\Sigma_{i j}$ are defined according to

$$
\Gamma_{i j} \doteq\left\langle\gamma_{i j}\right\rangle \quad \Sigma_{i j} \doteq\left\langle\sigma_{i j}\right\rangle \text {, }
$$


then the energetic macro-homogeneity Hill-Mandel condition is satisfied if an adequate set of boundary conditions ${ }^{3,58,145}$, either kinematic, static or periodic, is adopted for the microstructural unit cell. The relationship between the stress and strain macro-fields is then given by

$$
\Sigma_{i j}=\hat{c}_{i j k l} \Gamma_{k l} \quad \text { or } \quad \Sigma=\hat{\mathbf{C}} \Gamma \quad \text { Voigt notation }
$$

where $\hat{c}_{i j k l}$ are components of the apparent overall constitutive tensor; the hat denotes apparent overall properties, to distinguish them from the microscopic grain properties appearing in Eq.(3). The previous relationship is the homogenized stressstrain equation and forms the basis of several homogenization procedures. To completely characterize $\hat{\mathbf{C}}$, a suitable set of linearly independent kinematic boundary conditions has to be used.

A brief overview of microstructural models set in a linear framework is given in the following. Kozaczek et al. ${ }^{52}$ used the finite element method (FEM) to analyze the stress distributions within two Alloy 600 polycrystalline unit cube microstructures, with 250 and 500 grains respectively. Each grain was given a random orientation in space, i.e. no crystallographic texture was assumed. Moreover, each grain was assumed with cubic symmetry, i.e. three independent elastic constants $C_{11}, C_{12}$ and $C_{44}$ were assigned. Each crystal was divided into tetrahedral volume elements with a common vertex at the grain seed. The values of the aggregate elastic constants, computed through homogenization using the three-dimensional microstructural model, were in good agreement with some analytical estimations. Biaxial stress and almost uniaxial stress were considered as boundary conditions for the unit cube, to mimic the environment present at the component surfaces, where the intergranular stress corrosion cracking takes place in steam generators components, which was the investigation motivation. MSC-Nastran was used as FE commercial package. A similar study, addressed at estimating local grain-boundary stress concentrations due to crystal orientation mismatch, as performed by Kumar et al. ${ }^{51}$.

Nygards and Gudmundson ${ }^{56}$ developed a three-dimensional polycrystalline microstructural model for the analysis of a two-phase ferrite/pearlite steel. The microstructure was generated through Voronoi tessellation, it was periodic and subjected to periodic boundary conditions. The modelling strategy was validated assessing the material homogenization in case of orthotropic elastic behavior of the constitutive grains. The two-phase steel was however simulated adopting a simplified elastic-plastic behavior for the constituent phases. The work showed that, using periodic constraints, microstructures with only 5 grains are able to provide representative aggregate stress-strain curves. A similar FE model was employed to investigate the minimum number of grains to be included in an RVE for materials with cubic symmetry ${ }^{57}$. It was concluded that the number of grains necessary to homogenize such materials depends on the anisotropy of the constituents and a relationship in terms of the Zener anisotropy ratio was given. In the two aforementioned studies ABAQUS was used as FE software package.

Zhao and Tyron ${ }^{59}$ generated a statistical volume element (SVE) for single- and 
multi-phase polycrystalline metallic materials, to perform a statistical analysis of the von Mises stress distribution within the model microstructure. Aggregates with 100, 150 and 200 grains were generated through a Voronoi tessellation algorithm. Each grain was assumed to be homogeneous and anisotropic. The FE analysis was carried out using MSC Patran/Nastran. A similar analysis was performed by Kamaya et al. ${ }^{62}$, who specifically focused on the analysis of intergranular grainboundary stresses, which have a direct effect on the initiation of stress corrosion cracking phenomena. They performed a FE analysis of a 100-grain Voronoi-type microstructure and carried out a statistical analysis of grain-boundary stresses. MSC Patran and ABAQUS were respectively used to generate the microstructure and analyze the stress state.

A copper polycrystalline microstructure, carefully reconstructed from serial sectioning and ESBD, was modelled using the FEM by Sumigawa et al. ${ }^{117}$, who investigated the formation of persistent slip bands (PSBs) close to the grain boundaries in high-cycle fatigue tests. They assumed anisotropic linear behavior for the individual grains and assigned the experimentally determined crystallographic orientation. They concluded recording the coincidence between grain boundary stress concentrations due to crystallographic misorientation and the experimentally observed PSBs. Specifically focused on the identification of the size of the representative volume element (RVE) in linear elastic randomly structured polycrystals, made up of cubicshape cubic-symmetry single crystals is the study by Ranganathan and OstojaStarzewski ${ }^{33}$. In this study, the numerical simulations were performed on simple morphologies, i.e. aggregates of cubic-shaped grains with cubic material symmetry randomly oriented in space. However, the aim of the study was mainly theoretical, and the numerical simulations were mainly used to assist in the formulation of a scaling function for this class of materials. However it was specified that, although the scope of the work was restricted to simple aggregates, the developed concepts could be generalized to other crystal shapes and classes as well as to scaling of other elastic/inelastic properties

Böhlke et al. used FEM to carry out computational material homogenization of polycrystalline materials. In Fritzen et al. ${ }^{79}$, they considered FCC polycrystals with cubic symmetry, namely copper, gold and nickel. Periodic Voronoi microstructures generated with a specifically developed mesh generator were used and the grains were subdivided into tetrahedral elements using the Tetgen library (http://tetgen.berlios.de/index.html). Given a certain number of grains $N_{g}$, they generated a certain number of microstructures with $N_{g}$ grains, and estimated the apparent properties for each microstructural realization through volume averages. Then, they averaged these computed values over the number of generated microstructures, to improve the homogenization numerical convergence. The obtained results were in good agreement with analytical results, and with experimental results for copper, also for relatively few grains, due the periodicity of the mesh. In Bohlke et al. ${ }^{140}$ they considered a dental alloy, mainly consisting of gold, and carried out the homogenization using a 3D FE model. Analytical, numerical and experi- 
mental results agreed, confirming the quality of the computational model. The FE software package ABAQUS was used for these investigations.

Benedetti and ALiabadi ${ }^{69}$ recently developed a three-dimensional grain-boundary formulation for the analysis polycrystalline microstructures. The micromorphology is generated through hardcore Voronoi tessellation and the individual grains are assumed to be homogeneous anisotropic domains with arbitrary space orientation. The peculiarity of the model consists in the use of boundary integral equations for the representation of the elastic problem for the individual grain, which allow to express the polycrystalline problem directly in terms of grain boundary displacements and tractions that, as seen, play an important role in the aggregate micromechanics. The classical multi-region boundary element method is the natural choice for the numerical solution of the problem, which has the advantage of requiring the meshing of the grain boundary only. Material homogenization studies, similar to those considered by Fritzen et al. ${ }^{79}$, were successfully performed. The technique was also successfully applied to microstructures with pores, modelled as missing grains ${ }^{141}$.

\subsection{Models including crystal plasticity}

Crystal plasticity theory describes, in a continuum framework, the plasticity mechanisms in single and aggregate crystals ${ }^{146,147}$. It is based on the description of the kinematics of plastic slip, which takes place anisotropically over a set of active slip crystallographic planes. The crystal plasticity finite element method (CPFEM), either for single crystals or for aggregates, is classically used ${ }^{28}: a$ ) to study the grain scale distribution and localization of strains, which often result in strongly heterogeneous plastic fields, due to lattice misorientation between adjacent grains ${ }^{148,149}$, which can give rise to microscopic damaging processes; $b$ ) to predict the texture evolution of polycrystalline solids subjected to deformation processes, i.e. the lattice reorientation of crystals caused by the deformation $(150,37,46 ; c)$ to assess the quality of homogenization schemes based on simplified assumptions ${ }^{53,151}$.

It has been observed ${ }^{152}$ that two kinds of CPFEM may be found in the literature: local scale and global scale simulations. In small scale applications, the grains are represented explicitly and the focus of the investigation is on the computational reconstruction of local inter- and intragranular fields ${ }^{45,53,153}$. In global scale applications, the microstructure is not represented explicitly and the focus is on the aggregate behavior. Each integration point of a macro-FE mesh represents a group of crystals, whose properties are described through homogenized laws describing interactions among crystals. The resulting polycrystalline behavior is used as a macroscopic constitutive law, but provides better predictions of the final mechanical properties of the sample ${ }^{37,49}$.

Some introductory reviews of CPFE models are given in some papers in the literature ${ }^{142,28}$. A recent comprehensive review has been given by Roters et al. ${ }^{17}$, who thoroughly discussed several aspects of crystal plasticity modelling, from the 
essential inclusion of dyadic kinematics to the formulation of various types of crystal level constitutive laws, in a broad framework of scientific and technological applications, devoting attention also to comparisons of the predictions with experiments. More than 400 bibliographic entries are discussed in the review. The scope here is much more limited and mainly focused on the use of crystal plasticity in the 3D modelling of polycrystalline aggregates at the mesoscale.

In its basic small strain configuration, crystal plasticity is introduced by means of the Schmid law (eq. 9) which relates the crystallographic slip rate $\dot{\gamma}^{\text {s }}$ of a system $s$ to the plastic strain rate $\underset{\sim}{\dot{\varepsilon}^{g}}$ in a grain $g$. The Schmid tensor ${\underset{\sim}{m}}^{\mathrm{s}}$ is defined from the slip planes normals $\underline{n}^{\mathrm{s}}$ and slip directions $\underline{l}^{\mathrm{s}}$ of the considered slip system family and with the considered crystallographic orientation. Slip occurs on a slip system provided that the shear stress $\tau^{\mathrm{S}}$ exerted on it overcomes a threshold, just as in classical plasticity. This effective shear stress is computed also from the Schmid tensor, as defined from eq. 10.

$$
\begin{gathered}
\dot{\sim}^{\mathrm{g}}=\sum_{s}{\underset{\sim}{m}}^{\mathrm{s}} \dot{\gamma}^{\mathrm{s}} \quad \text { where }{\underset{\sim}{m^{\mathrm{s}}}}^{\mathrm{s}}=\frac{1}{2}\left(\underline{n}^{\mathrm{s}} \otimes \underline{l}^{\mathrm{s}}+\underline{l}^{\mathrm{s}} \otimes \underline{n}^{\mathrm{s}}\right) \\
\tau^{\mathrm{s}}=\sigma:{\underset{\sim}{m}}^{\mathrm{s}}
\end{gathered}
$$

From that point, crystal plasticity may be classified into two main categories: ratedependant or rate-independant. The challenge in this latter case is to deal numerically with the determination of the active slip system which happens as a singularity in the numerical process. To avoid any singularity affecting numerical convergence, it is common to deal with a viscoplastic framework, within which parameters can be tuned to inhibit viscous effects if needed. Models can then be classified into two other categories: those where the effective shear stress on a slip system is determined from phenomenological hardening laws mimicking the increase in dislocation density and those, more physically consistent, where dislocation densities are explicitly accounted for. The model of Asaro ${ }^{146}$ and its extension by Méric and Cailletaud 154 to a combination of isotropic and kinematic non linear hardening of the slip systems provide common examples of this phenomenological approach: a system is active provided that $\tau^{\mathrm{s}}$ is greater than a state of stress which is determined from a combination of isotropic and kinematic non linear hardening on the slip systems and these hardening components obey non linear evolution laws of the Chaboche type. In a typical dislocation-density based model (see e.g. ${ }^{65}$ ), $\dot{\gamma}^{\mathrm{s}}$ is determined from a flow rule of the type of eq 11, as a function of $\tau_{s}$ and temperature; the hardening rule for $\tau^{\mathrm{S}}$ accounts for the dislocation densities $\rho^{s}$ in all the slip systems and these dislocation densities are determined according to the slip rate $\dot{\gamma}^{\mathrm{s}}$ and the mean free path of a dislocation $D_{\text {path }}$ by means of an evolution law as illustrated in eq 12 .

$$
\begin{gathered}
\dot{\gamma}^{\mathrm{s}}=f\left(\tau_{s}, T\right) \\
\tau_{s}=f\left(\rho^{s}\right) \quad \dot{\rho}^{s}=f\left(\dot{\gamma}^{\mathrm{s}}, D_{\text {path }}, \rho^{s}\right)
\end{gathered}
$$


Several three-dimensional finite element models incorporating crystal plasticity laws were initially developed to assess texture development. In several studies ${ }^{37,39,40,42,44}$, the assumed morphology was quite simple, and often coarse meshes, with an individual grain represented with a single finite element, were employed. Although the simple representation of the microstructure, these models were quite accurate in predicting texture evolution.

The local and global response of elastic and visco-plastic anisotropic polycrystals has been investigated by Lebensohn ${ }^{155}$ adapting a Fast Fourier Transform (FFT) technique previously developed by Moulinec and Suquet ${ }^{156}$. A simplified cubic-grain morphology was used to represent the polycrystal. The FFT formulation is restricted to RVEs under periodic boundary conditions and, for these cases, its performance was shown to be largely superior that of the FEM calculation for problems of the same size. The model was used to predict both local and overall textures of a single phase fcc polycrystal deformed in plane-strain compression. It predicted intragranular misorientation and subgrain formation. The texture development predicted with the FFT model was in good agreement with typical fcc textures, measured for the same deformation.

Delannay et al. ${ }^{49}$ focused on the prediction of texture development and microscopic strain heterogeneity in cold-rolled ULC steel and in multiphase steel under uniaxial tension. They represented the microstructure as an aggregate of cubes or truncated octahedrons, meshed as a finite element mesh and loaded with periodic boundary conditions. This work belongs to a set of investigations in which the microstructure is not explicitly modelled, but it is assessed on a statistical basis. The model microstructure resembles the real microstructure in terms of texture, volume fraction of phases, grain shape, grain size distribution, etc. and, if a sufficient number of grains is contained in the model microstructure, then it is statistically representative of the sample under consideration and numerical predictions may be validated against experimental measurement of the texture development ${ }^{37,44,157,47,158}$. A simplified implementation of crystal plasticity, in which elastic strains are considered infinitesimal, was used in the work. The use of truncated octahedrons, instead of bricks, was suggested to overcome the underestimation of strain heterogeneity between hard austenite grains and softer surrounding grains made of ferrite and bainite. It was found that this assumption yielded improved predictions compared to those with cuboidal grains.

Zhang et al. ${ }^{159}$ developed and implemented a 3D microstructure-based constitutive model for monotonic and cyclic deformation of duplex Ti-6Al-4V. Individual grains were represented as identical cubes in the FE mesh and the model was calibrated to adequately represent the microstructures of the tested material. Equivalent orientations with similar probability density distributions of the crystallographic orientations were assigned to the finite element mesh. Crystal plasticity laws were applied to both primary a phase and lamellar colony via ABAQUS implementation. For a given Ti6 Al4V alloy, it was shown that the mechanical deformation could be correctly predicted by conducting the FE simulations the proposed material modeling. 
Effects of microstructural features were examined and compared well the experimental data, in terms of influence on the material yield strength.

Although models with simplified representation of the microstructure were able to produce acceptable predictions of the texture evolution, the accurate representation of local micro stress/strain gradient, especially close to the grain boundaries, required more a realistic representation of the microstructure. One of the first attempts at simulating a fully three-dimensional microstructure with discretization of individual grains was done by Sarma et al. ${ }^{91}$, who also combined the deformation model with a recovery and recrystallization model. It was assumed that elastic deformations are negligibly small, and that deformation occurs by slip dominated plastic flow. Due to the limited modes of slip deformation, the crystals must rotate to accommodate arbitrary deformations. The preferred reorientation of crystals leads to texture development, modeled using crystal plasticity constitutive laws.

Quilici and Cailletaud ${ }^{160}$ developed a crystal plasticity finite element framework, that was subsequently used for a detailed analysis of the intergranular and transgranular stress/strain fields within 3D polycrystalline aggregates in the small strains regime ${ }^{53,54}$. Voronoi polyhedra were used to represent the microstructure and a specific crystallographic constitutive model, accounting for visco-plasticity, isotropic and kinematic hardening was employed. Aggregates up to 200 grains were analyzed and attention was devoted to the high computational requirements of this $3 \mathrm{D}$ computations. The FETI method ${ }^{161}$ was employed in conjunction with high performance computing (PVM and MPI) to tackle the problem. The same strategy was later employed by Diard et al. ${ }^{60}$, who focused on a detailed analysis of micro plasticity in hexagonal close-packed materials, with the declared aim of representing an "as realistic as possible" microstructure. In these studies, reference is made to the possibility of investigation damage initiation mechanisms, thorough an accurate modelling of the microstructure.

Choi ${ }^{162}$ investigated the the plane strain compression of polycrystalline interstitial free steels with CP FEM, using a simplified quasi 3-D geometric mesh. The simulations provided a quantitative assessment of the orientation-dependent stored energy in cold-rolled IF steels. Sarma and Radhakrishnan ${ }^{163}$ applied CPFEM for studying the hot deformation of grains in polycrystalline aluminum. They report that, due to the time-consuming nature of such simulations, it was possible to consider only a limited number of specially constructed microstructures. The simulations were able to capture many experimentally observed features, then providing a useful tool for investigating the cube deformation and recrystallization textures during cold and hot deformation of aluminum alloys. Zhang et al ${ }^{61}$ investigated the role of micro-plasticity in the response of polycrystalline hexagonal ceramics under uniaxial-strain shock compression. They developed a 3D Voronoi finite element (ABAQUS/Standard) polycrystal model for the analysis of heterogeneous microplasticity, with compression-dependent anisotropic elasticity and rate-dependent crystal plasticity and analyzed aggregates containing 1001000 hexagonal crystals, concluding that a 600 -grain Voronoi polycrystal provides a statistically accurate 
model. The possibility that micro-plasticity due to basal slip $\langle 11 \overline{2} 0\rangle\{0001\}$ is the dominant mechanism governing the inelastic response of polycrystalline hexagonal ceramics under uniaxial-strain shock compression was investigated. The crystal elasticity parameters were determined from the known material properties of $\alpha-6 \mathrm{H} \mathrm{SiC}$. The crystal plasticity parameters were chosen so that the resulting plastic response was essentially non-hardening and very weakly rate-sensitive. The results showed that the assumption of basal-slip only micro-plasticity does not cause physically unreasonable micro-mechanical states, with the computed volume-averaged response matching the experimental data well up to a longitudinal stress of $20 \mathrm{GPa}$. Among other conclusions, the authors interestingly observed that the micro-plastic process generates micro-mechanical heterogeneities of such intensity to justify the possibility of micro-cracking initiation at micro-plasticity generated stress concentrations, if micro-cracking is also a viable mechanism in shock-compressed ceramics.

An interesting application of the crystal plasticity theory in a 3D finite element framework can be found in You et al. ${ }^{124}$, where the deformation of a thin 316LVM stainless steel strut, often used for cardiovascular stents, is analyzed. The models were obtained starting from in situ deformation experiments performed using SEM, digital photogrammetry and OIM. An idealized prismatic grain model and a fully $3 \mathrm{D}$ model were analyzed: results showed that the models gave a very good prediction of the global stressstrain curve, with the fully 3D model being slightly more accurate. The models produced similar maps of local surface strain components, when compared with experimental maps, and predicted the grain level active slip systems and the evolution of the active systems during deformation. The prediction of grain reorientation during deformation and the development of discrete deformation bands within the grains was less accurate. The authors suggested addressing these issues enhancing the accuracy of the material strain hardening description introducing a length scale representation in a strain gradient plasticity framework. However, this was not the most relevant aspect in the considered application, were the reconstruction of the global stress-strain curve was the most important objective.

Zeghadi et al. ${ }^{28}$ performed, in a crystal plasticity finite element framework, a statistical analysis on the behavior of several different 3D morphologies generated starting from the same 2D surface. Aggregates with around 40 grains were considered and the plastic strain fields on the reference 2D surface, induced by different internal 3D morphologies of the assessed specimen, were investigated and it was found that, in the case of copper grains, large fluctuations in the equivalent plastic slip level are induced by different internal 3D morphologies, under the same external loading conditions. The conclusions of the study advocate the need of full 3D studies over simplified pseudo 3D (or 2D columnar) studies. Similar studies, focused on the comparison between experimental and computational results, with the aim of identifying possible sources of error and better calibration microstructural constitutive parameters were performed by Héripré et al. ${ }^{164}$ and Musienko et al. ${ }^{120}$. Musienko et al. ${ }^{120}$ 
analyzed an experimentally reconstructed copper specimen with approximately 100 grains. Crystal plasticity finite elements were used and the results of fully 3D and columnar 2D (pseudo-3D) simulations were compared in order to assess the bias introduced by the simplified mesh generation. It was found that the computational results of the full 3D mesh were in good agreement with the experimental data, while only some traces of the experimental behavior were retained by the extended 2D simulations. Héripré et al. ${ }^{164}$ applied a similar method to zirconium and titanium aluminide alloys, employed in the nuclear and aeronautic industries, in order to improve the understanding of their micro-mechanical behaviour.

Zhao et al. ${ }^{165}$ used the finite element crystal plasticity framework to investigate strain localization and surface roughening observed in experiments in polycrystals, to get insight into grain-scale roughening mechanisms and to assess the robustness of numerical models used in the prediction of local surface displacements. With this motivation, they conducted a series of uniaxial tension tests using EBSD, digital image correlation and surface roughening measurements on a three-dimensional (quasi 2D) dog bone oligocrystal sample. On the basis of their simulations, they drew some conclusions and pointed out some limitations of crystal plasticity modelling, related to its continuum nature, in addressing some experimentally observed micro-deformation patterns.

A crystal plasticity framework for spatially controlled bainitic steels microstructures has been developed by OsipovAl2008 ${ }^{63}$, with a view towards the prediction of brittle failures in nuclear power plants components. The influence of morphology, local texture in the phases, mesh type and mesh size was investigated, and special attention was devoted to the comparison between bainitic microstructures versus an equiaxed ones. A relevant spurious effect has been found with meshes not conforming the bainitic packets boundaries and spanning more phases: an increase in the local maximum stress has been observed, concluding that multi-phase elements do not provide reliable local stresses and should be avoided in such applications. Among the conclusions, an interesting suggestion was given on the suitable number of grains/finite elements to be considered in microstructural failure initiation modelling, taking into account the possibility of extreme intergranular events that may enable damage initiation.

Since then, the approach has been extended to large deformation computations with dislocation-density based constitutive laws presented in ${ }^{65}$. N'Guyen et al. ${ }^{32}$ presents a short overview of this approach with an application aiming at predicting cleavage fracture of the polycrystal from a multiple micro-cracks scenario. For this application, all the characteristics of the approach presented in ${ }^{63,64,65}$ have been gathered and complemented by the use of a large scale and statistically representative microstructure of bainite polycrystal enabling the prediction of rare cleavage micro-cracks events. Fig.3 presents some of the results of this study. Barbe et al. ${ }^{166}$ studied the localization of plastic strains in the elasto-plastic strain fields of a highresolution 3D polycrystalline representative volume element, considering that such information is not easily experimentally accessible and that it can however be an 


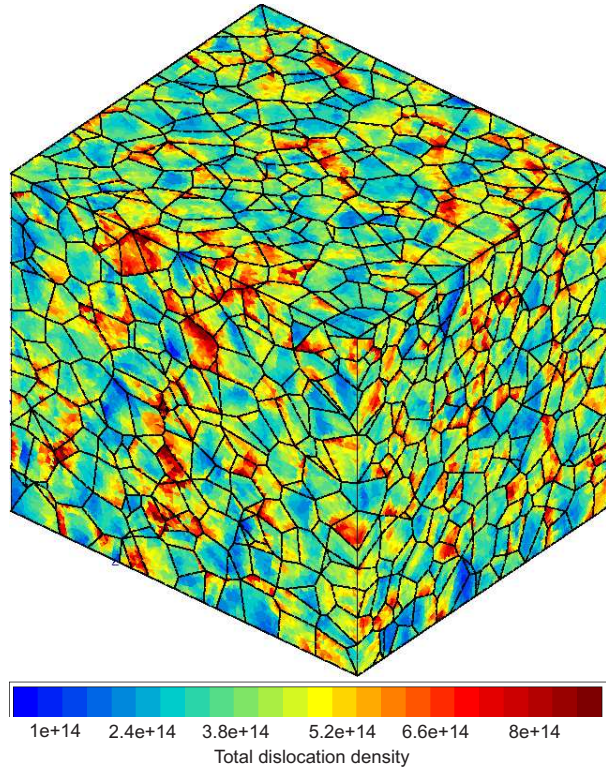

(a)

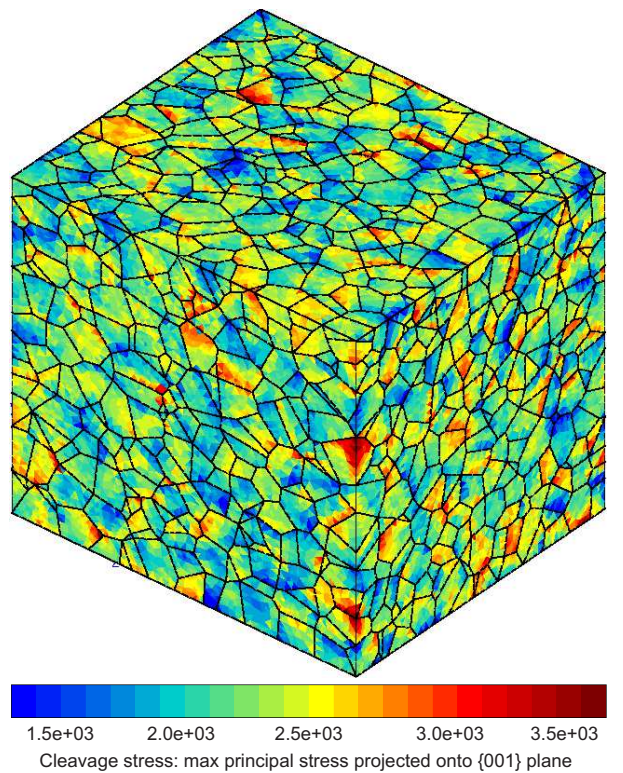

(b)

Fig. 3. Bainite polycrystal subjected to $10 \%$ plane strain tension with triaxiality $=2.4096$ Voronoi cells represent the bainitic packets of 80 primary austenite grains. The contour plots of (a) the total dislocation density and (b) the cleavage stress illustrate how differently can transgranular localization take place according to the considered field variable. After ${ }^{32}$.

important factor for the representativity of a microstructural morphology. A modelling strategy similar to that used in Barbe et al. ${ }^{53}$ was used here and the 3D distribution of highest strains was analyzed with considering the point-to-point covariance of segmented strain field. The propensity for strains to localize with the same probability along bands large 2 or 3 mean grain size oriented at around $45^{\circ}$ with respect to the tensile direction was shown. Since experimental characterization of these features is still today difficult or impossible, the work constitutes the first step toward such detailed and systematic 3D characterization.

In the work by Resk et al. ${ }^{142}$, which is mainly devoted to the development of a meshing and remeshing strategy for polycrystalline microstructures under large strains, the rolling of a 500-grains aggregate subjected to more than $90 \%$ thickness reduction has been simulated. Better texture evolution predictions with respect to standard polycrystal plasticity models such as the Taylor model is obtained. The investigation shows that CPFEM, combined with appropriate modelling and computational techniques, also in terms of HPC, offers a viable alternative to study the micro-plasticity in aggregates of several tens or hundreds or even thousands of grains. The authors also point out how classical crystal plasticity models are not able to address complex microstructural evolutions at the sub-grain scale or near grain boundaries and the need for physically based constitutive models is advocated. 
Barbe and Quey ${ }^{55}$ presented a numerical CPFE model for 3Dpolycrystalline microstructures including polycrystal-to-polycrystal diffusive transformations. They implemented the main micro-scale features of the kinetics of diffusive transformations in actual steels: nucleation and growth at preferential sites of the microstructure with laws depending on time. The elasto-plastic interactions between parent and growing grains is solved by the CPFE method at the intra-crystalline scale of a representative volume element. On the basis of simulations results, the authors pose some interesting fundamental questions about the experimental identification of material parameters. Moreover the study calls points out the difficulty of experimental measurements of some parameters affecting the transformation process, such as nucleation rate, distribution of nucleation sites, growth rate and suggest to address these issues through sensitivity analysis or physically based modelling of microstructural evolution at the local scale of few grains.

Schneider et al. investigated the mechanical behavior of alphaFe-Cu composites, with experimentally reconstructed axial-symmetric microstructures ${ }^{167}$ and artificially generated Poisson-Voronoi microstructures ${ }^{168}$, under large plastic deformations in simple tension and compression, using 3D FE simulations (ABAQUS) with an elasto-visco-plastic material model. Several different composites have been investigated in detail and the local deformations, the flow behavior, the texture, and the distribution of the strains were considered, in order to reveal the effects of local events on the global behavior and the dependence upon the phase distribution.

Li et al. ${ }^{169}$ used CPFEM to simulate the behavior of aluminum alloy AA2024 under nano-indentation. The morphology was generated by Voronoi algorithms, the grain orientations were imported from EBSD and the model parameters were calibrated fitting the stressstrain curves obtained from some tensile tests performed at different angles with respect to the rolling direction. The simulations showed that the local deformation, at the indentation area, strongly depends on the grain properties and matched the experimental data very well, in terms of simulated forcedisplacement curves and indentation moduli. The effect of the microstructure on the macroscopic indentation curves was demonstrated, observing also the effect of low and high grain boundaries close to the indentation zone. The study was the first CP investigation on the behavior of individual grains, in polycrystalline aggregates, under nano-indentation and provided detailed three-dimensional information not easily obtainable from experiments.

\subsection{Modelling microstructural damage}

The possibility of modelling material microstructural damage initiation, coalescence and evolution and capturing the process by which micro damage, voids and cracks evolve into macro damage or cracks, capable of affecting the functionality and/or safety of engineering structures, is attracting considerable attention, due also to the development of more affordable computational capability. The aim of such in- 
vestigations is to understand how macro damage or cracks are nucleated. Ideally, such information would complement that provided by classical continuum damage and fracture mechanics, which study how damage or cracks, whose presence is a priori assumed at some critical locations, propagate in a structure subjected to given loads. In other words, while classical approaches assume the presence of preexisting damage and study how it propagates at a continuum level, microstructural approaches investigate how damage is nucleated at the microscale and how it migrates to the macroscale. It is clear that, once some kind of macroscopic damage is initiated, it may in turn affect the initiation of new damage, favoring and accelerating those microscopic processes that led to the nucleation of damage in first place. In this sense damage is a multiscale phenomenon and it requires a multiscale approach for understanding how micro and macro processes interact ${ }^{170,171,172,173}$. In this framework, the capability of modelling the microstructure and its evolution is a fundamental tool, with the potential of making more predictable properties such as strength, toughness, ductility, fatigue life, etc. which are of great relevance to designers.

This section is dedicated to a brief survey of three dimensional computational approaches to damage and fracture in polycrystalline aggregates. Comprehensive recent reviews have been given by other authors, such as Crocker et al. ${ }^{22}$ or Bieler et al. ${ }^{174}$. In this short survey, the focus is on works considering three-dimensional models.

The initiation and evolution of damage in polycrystalline microstructures is a complex phenomenon that can assume different features and is affected by several factors. At temperatures below $0.3 T_{\text {melting }}$, the main fracture mechanisms in polycrystalline aggregates $\operatorname{are}^{22}$ :

- brittle intergranular fracture, in which the micro cracks propagate along the grain boundaries;

- brittle transgranular or cleavage fracture, where the cracks propagate through the grains along some specific crystallographic planes;

- ductile fracture, which originates from the nucleation and coalescence of micro voids initiating at second phase impurities.

At higher temperatures, creep becomes more important.

Such complexity makes the computational modelling of fracture in polycrystalline microstructures particularly challenging as it is reflected by the lack of comprehensive tools able to address simultaneously all the possible damaging mechanisms. A fundamental aspect of polycrystalline materials, which has a strong influence on crack propagation and should be retained in any computational representation, is the microstructural heterogeneity in terms of morphology and crystallographic orientation, which is responsible for the strain and stress concentrations that arise when the microstructure tries to accommodate a macroscopic deformation: if local strains can accommodate the geometry changes, no damage is initiated, whereas damage may arise where insufficient strain or shape accommodation occurs. Crys- 
tal plasticity, often used in conjunction with the finite element $\operatorname{method}^{175,17}$, is a general framework accounting for such heterogeneity ${ }^{174}$. On the other hand, homogenization models, due to their inherent features and scope, are not able to provide a detailed local representation, and are then not suitable for damage characterization, while atomistic or discrete dislocation density models can deal with volumes too small to cope with the simulation of mesoscale damage nucleation. In other words, although damage nucleation is undoubtedly related to atomistic phenomena, the factors affecting it appear to originate mainly at a mesoscopic (grain) scale, to such an extent that the grain scale appears as the most suitable to address its study.

An important aspect deriving from microstructural heterogeneity is the presence of intergranular interfaces, which play a fundamental role in the initiation of damage. Some studies, in the framework of grain boundary engineering, evidenced the dependence of material's strength and damage nucleation resistance on the grain boundary character, which depends on the interfacial structure ${ }^{176,177,178,5,179,180,181,182}$. According to this paradigm, the grain boundary cohesive strength, and then quality of the boundary, depends on its atomic packing efficiency. In turn, the material properties, especially in terms of damage nucleation propensity, depend on this boundary quality. A popular approach for taking into account these energy based considerations and modelling intergranular damage initiation and evolution is based on the use of cohesive laws $183,184,185,186,187$. Cohesive mixed-mode traction-separation laws express interfacial tractions $\mathbf{t}$ as functions of the interfacial displacement opening and sliding $\delta \mathbf{u}$, through relationships of the form

$$
\mathbf{t}=\mathbf{K}(d) \delta \mathbf{u}
$$

that in general may embody the dependence on a scalar damage parameter $0 \leq d \leq$ 1, varying between 0 (pristine state) and 1 (failed or cracked interface). In Eq.(13), the interface cohesive energy is the work required to take the interface from a pristine to a failed state.

Cohesive laws have been employed, together with finite elements, in several threedimensional studies about polycrystalline damage. Drawing on experiments and atomistic simulations present in the literature, Wei and Anand ${ }^{188}$ observed that, when the grain-size is under $\approx 100 \mathrm{~nm}$, grain-boundary slip and separation start having a major role in the overall inelastic response of a polycrystalline material, while dislocation activity within the grain interiors becomes more difficult. To model these effects, they coupled an isothermal, rate-independent crystal-plasticity model for the bulk grains with an isothermal, rate-independent elasticplastic grainboundary interface cohesive model, accounting for both reversible elastic and irreversible inelastic sliding-separation deformations at the grain boundaries. The model was then applied to the analysis of the deformation and fracture response of nano-crystalline nickel. Several 3D columnar aggregates were analyzed with a FE ABAQUS implementation. The simulations reproduced the experimental macroscopic tensile stressstrain curves and the dominant microstructural fracture mecha- 
nisms. The effects of the variation of the grain-size on the macroscopic stressstrain curve were also investigated. Results of models including crystal plasticity were compared with results of models retaining only the elastic behavior of the grains. The authors showed that the macroscopic nonlinearity in the stressstrain response is mainly due to the behavior of the grain boundaries, while only limited plastic deformation of the interior grains was observed. The competition between grainboundary and grain interiors deformation was shown to determine the observed macroscopic stressstrain response, and the overall ductility. Also the fracture behavior of a notched specimen was investigated. The authors observed that their model could be suitable to analyze inelastic deformation and fracture of materials with little or no transgranular plasticity, such as ceramics and rock-like materials, where the pseudo-plasticity and non-linear stressstrain curves arise primarily from grain boundary separation and sliding.

The damage and cracking behavior of polycrystalline specimens subjected to quasi static loads has been investigated by Luther and Könke ${ }^{66}$, who focused on the development of a modified Voronoi algorithm to improve the approximation of grain size distribution in artificially generated microstructures. The intergranular interfaces were represented with a cohesive zone model, to simulate crack propagation along grain boundaries. The authors chose a traction separation law with a sharp peak and an exponential decohesion path to simulate brittle intergranular fracture; they observed how the choice of the cohesive parameters deserves careful consideration, as it remarkably affects the aggregate mechanical behavior. Their tests showed how the grain size distribution influences the sample effective tensile strength. The model with a classical Voronoi geometry was also employed for simulating a small 3D sample, although the computational limitations imposed by the increased number of degrees of freedom were pointed out.

Benedetti and Aliabadi ${ }^{70}$ developed a 3D grain boundary integral model for intergranular degradation and failure of polycrystalline microstructures subjected to quasi static load conditions. The grains were represented as elastic anisotropic regions and boundary integral equations were used to represent the elastic problem for the individual grains. No plasticity was assumed for the grains. The interface was conveniently modelled using extrinsic mixed-mode bi-linear cohesive laws, incorporating a damage parameter expressing the irreversible intergranular degradation process. When an interface is completely damaged a micro-crack is introduced in the simulation and a frictional contact analysis, taking into account the possibility of separation or contact between the crack surfaces, was used to further follow the microstructural evolution. The model offers the advantage of simpler data preparation with respect to the more popular FE analysis, as only meshing of the intergranular interfaces is required in the problem. The classical boundary element method is used for the numerical discretization. Several micromechanical analysis were performed and the method appear promising for future multiscale applications.

The above works focused mainly on quasi static intergranular failure, employing cohesive laws to express the progressive failure of the interface. Other topics of great 
relevance in this framework are intergranular stress corrosion cracking (IGSCC) and intergranular failure due to fatigue loads. These topics have been addressed at the grain scale level, either employing cohesive relationships or continuum damage mechanics approaches.

IGSCC is a complex phenomenon in which the nucleation and propagation of damage is affected by the interplay of grain boundary features (precipitates, boundary character, etc.), environment and loading conditions. Several models have been proposed in the literature, but a unified treatment has not been attained, and it is maybe not attainable, due to the specificity of the material/environment/load set, which may change from application to application. Jivkov et al. ${ }^{48}$ developed a computational 3D mechanical model for IGSCC based on the regular representation of the microstructure with truncated octahedra. The model was intended as an advancement from percolation-like models ${ }^{176,189}$, by inclusion of mechanical effects that allow the assessment of the influence of branching and bridging on the crack driving force. Grain boundaries are categorized either as beneficial or detrimental to fracture and, based on FE stress calculations, the model provides a simulation of crack evolution, where the crack advance depends upon the actual mechanical conditions at the crack tip. Results for cracks propagation and coalescence were presented and they were consistent with experimental observations, demonstrating the capability of simulating the crack bridging in the crack wake as well as crack coalescence.

Kamaya and Itakura ${ }^{190}$ studied the initiation and propagation of the microcracks using concepts of continuum damage mechanics in a FE framework. A 6000-grain three-dimensional polycrystalline body was generated by Voronoi tessellation. Each grain was decomposed into tetrahedral elements and different grains were bonded placing thin intergranular elements with assigned stiffness between adjacent grains. Cracks were assumed to grow along the grain boundaries and the grain boundaries normal stress was used for crack initiation and growth. Damage was assumed to accumulate at grain boundaries and a crack is initiated, setting the elastic constants of the intergranular elements, when the accumulated damage exceeds the resistance to cracking. The rate of accumulation of damage depends on the stress acting on the grain boundary. The simulation reproduced the changes in the number of cracks and sum of crack lengths obtained from experiments. Simonovski and Cizelj ${ }^{19}$ studied IGSCC in a section of a wire of AISI 302 stainless steel. The microstructure was experimentally reconstructed by DCT and the analysis was conducted by FEM, with grains assumed as isotropic and elastic. Cohesive laws were used for modelling the intergranular interfaces, with zero thickness finite elements, and the grain boundaries were classified either as resistant, modeled without damage evolution, or susceptible, modeled with damage initiation and evolution. A field variable was defined to model the corrosive environment. The model was afterwards extended to include crystal plasticity ${ }^{20,30}$.

Musienko and Cailletaud ${ }^{67}$ developed a comprehensive computational framework 
for the analysis of iodine-assisted SCC in Zircaloy tubes used in nuclear power plants. The model takes into account crystal plasticity, environmental effects and the competition between inter and transgranular failure. The microstructure was generated through Voronoi tessellation, while the grain-boundaries were obtained by shrinking the grains, so to generate intergranular interstitia to be afterwards meshed through suitable 2D or 3D intergranular finite elements with their own constitutive behavior. The grains interior was modelled with a crystal visco-plastic model $^{154,191,192}$, extended to take into account the possibility of transgranular cleavage. Moreover, grain boundary elements were used to take into account the possibility of intergranular damage, opening and sliding. The damage was taken into account through a scalar damage quantity, following a classical continuum damage mechanics approach, and the laws governing the damage kinematics were given. In other words, failure is modelled combining a damage mechanics approach for intergranular fracture (generated by cavity nucleation and growth) and a visco-plastic law with softening (negative hardening) for cleavage. The environmental effects, namely the presence of iodine, were simulated through the classical diffusion Fick's law, and a weak coupling between diffusivity coefficients and damage has been implemented. The comprehensive computational tool was then applied to several 2D and some 3D cases, to assess accuracy and numerical stability and promising results and a good correlation with experimental studies were obtained. However, only one $3 \mathrm{D}$ case was reported and only intergranular failure was allowed in the 3D case, apparently due to the high computational cost of a fully coupled 3D analysis. As future development, the authors suggested the inclusion of additional chemical or electrochemical parameters for a better characterization of environmental effects. Intergranular fatigue damage in polycrystalline specimens was investigated by Bomidi et al. ${ }^{21}$, who presented a 3D FE model to account for topological randomness in material microstructures for micro-electromechanical systems (MEMS) devices. The material topology is generated via 3D Voronoi tessellations, while progressive fatigue material degradation is modelled within a continuum damage mechanics framework. A debonding criterion was developed for both intergranular crack initiation and propagation stages. The fatigue damage model was then used to investigate the effects of microstructure on the fatigue life of cantilever MEMS devices, focusing on morphological and material randomness, as well as on the effects of defects and internal voids. Obtained stresslife results, damage progression and the overall crack pattern were consistent with experimental or empirical observations. The model was also used to identify geometric modifications beneficial to the fatigue life of micro cantilever beams subjected to bending. The microstructural topology had been previously explicitly modelled also by Weinzapfel et al. ${ }^{68}$, to investigate subsurface stresses in Hertzian contacts and provide a better understanding of rolling contact fatigue. 3D Voronoi tessellations were used in the study and an ABAQUS finite element analysis was performed. Internal stresses for the rolling contact resulted consistent with analytical solutions and the effects of the micromorphology on the micro-stress distribution was assessed. 
In some studies, the nucleation of microstructural cracks bas been correlated with the formation of persistent slip bands, due to the accumulation of plastic deformation. In this context, CPFE is a natural tool of investigation. Polycrystal plasticity was used by Manonukul and Dunne ${ }^{193}$ to study both low and high cycle fatigue (LCF \& HCF) in a nickel based alloy. They modelled a representative region of the material, around 60 grains, taking into account grain morphology and crystallographic orientation. The model was capable of predicting correctly the slip accumulation and a fatigue crack initiation criterion was proposed, based simply on a critical accumulated slip: crack initiation occurs, when critical slip is achieved within the microstructure. The model replicated several experimentally observed phenomena and it is based on two material properties: the critical resolved shear stress and the critical accumulated slip. A simple threedimensional extension of the model provided an estimate of the fatigue limit for HCF crack initiation in the nickel-based alloy $C 263$. In ${ }^{194}$, LCF tests were carried out on a pseudo-3D (2D columnar) polycrystalline nickel-base alloy specimen. The microstructure was reconstructed using EBSD and polycrystal plasticity analyses mimicking the experimental conditions were performed. It was demonstrated that fatigue crack nucleation and growth depended crucially on features such as crystallographic orientation, grain boundaries, triple points and inclusions. It was shown that crack nucleation occurs at locations where localised slip banding was predicted to develop by the crystal plasticity FE. Sinha and Ghosh ${ }^{195}$ focused on the development of an experimentally based CPFE micromechanical model ${ }^{196,197}$ for predicting the nucleation of fatigue cracks and total life in high strength low alloy (HSLA) steels subjected to cyclic loading over a prolonged duration with high mean stress. In these conditions these materials experience a failure mode known as cyclic ratcheting, due to the local accumulation of plastic strain, leading to nucleation and growth of subsequent cracks by void growth and coalescence. The strain accumulation, orientation and anisotropy dependence must be represented by the microstructural FEM model. A realistic prediction of nucleation life in ratcheting must refer to the estimation of local strains. A simplified grain morphology (unit cube divided into a mesh 1728 8-noded brick elements in ABAQUS-Standard) was used for representing the polycrystal and OIM information was incorporated in the CPFEM, to account for crystallographic orientation and misorientation distributions. Calibrated elasticity and crystal plasticity material parameters for individual crystals were estimated. Stress-controlled cyclic deformation conditions were simulated and a maximum local plastic strain-based nucleation model was proposed for predicting ratcheting life. The model was found to provide results consistent with experimental observations. It is worth noting, however, that the actual initiation of a dominant crack was not explicitly modelled, and the onset of dominant nucleation was assumed to occur at a critical plastic strain estimated on the basis of experimental observations. Also in this work the authors observed that the crystal plasticity computations for cyclic loading were quite expensive, and simulating the entire life for the microstructure is prohibitive. Consequently, simulations were run until stabilization of the ratcheting curve was 
reached.

Although the grain boundary engineering paradigm, in the investigation about damage nucleation, has focused mainly on the inherent properties of interfaces, recent studies, in the crystal plasticity framework, have started pointing out the importance of the interaction between dislocation activity in the interior grains and grain boundaries ${ }^{198}$. Querin et al. ${ }^{199}$ investigated the formation of micro voids at certain grain boundary triple point junctions in thin sheets of rolled aluminum alloy AA6022, subjected to monotonic tensile loading, using CPFEM with an experimentally reconstructed microstructure. Bieler et al. ${ }^{174}$ provided a careful assessment of the state of the art about damage nucleation in polycrystalline microstructures and, on the basis of experimental and computational CP-FEM modelling of TiAl aggregates, concluded that: a) large local strains prevent damage nucleation providing geometric accommodation that relieves local conditions favorable to damage; b) damage nucleates from imperfect slip transfer through grain boundaries, which leaves residual dislocation content in the boundary plane; $c$ ) damage nucleates at grain boundaries where unfavorable slip interactions weaken the boundary itself; d) slip directions are more influential on damage nucleation than slip planes. In their study, they advocate the need of grain boundary damage nucleation criteria incorporating: $a$ ) the orientations of crystals on either side of the interface; $b$ ) the boundary orientation and structure, related to the boundary energy; c) the activated deformation systems on either side of the boundary; $d$ ) the stressstrain gradient history in the grains on either side of an interface. They observed, on the other hand, that research considering all the mentioned factors is rare.

Ductile damage evolution at the grain scale was investigated by Lebensohn et al. ${ }^{200}$, who developed their FFT technique ${ }^{155}$ to study void growth in three-dimensional microstructures. They merged polycrystal plasticity and dilatational plasticity to compute growth of intergranular voids in porous polycrystalline materials. The formulation was applied to a fcc polycrystal with uniform texture and intergranular cavities, and to a porous material with homogenous isotropic matrix and identical initial porosity distribution. Vose et al. ${ }^{201}$ proposed a micromechanical model of creep induced grain boundary damage and simulated creep damage in a polycrystal with finite element analysis. Grain boundary cavitation and sliding were considered according to a micro-mechanically motivated cohesive zone model, while creep deformation of the grains was described with crystal plasticity. The model enabled the simulation of grain boundary creep damage in polycrystalline structures and it can be applied either to idealized polycrystalline structures or to real grain structures of miniature creep specimens. The cohesive law employed to model creep, however, requires careful experimental calibration, due to the large number of parameters entering it. 


\section{Multiscale Modelling of polycrystalline solids}

The explicit simulation of the polycrystalline microstructure and its evolution finds a remarkable application in the multiscale analysis of solids, in which a considered component is analyzed simultaneously at the component level, in which the load history is defined, and at the grain scale level, which provides the constitutive material evolution. The term multiscale can assume a variety of meanings ${ }^{170,171,172,173}$ : however here we focus on simulations involving two spatial scales, the continuum level and the grain scale level. The objective of these studies is the analysis of both the behavior of the macro-component and the processes happening at the microscale during the loading history. The multiscale analysis becomes particularly useful when, during the loading history, the microstructure undergoes transformations or damage, so that a simple constitutive model assumed at the macro-level could not be used to simulate the behavior of the structure, see for example Fig.4. It is worth mentioning that, due to the enormous computational effort required by a full three-dimensional multiscale analysis, which usually involves the simultaneous solution of several three-dimensional non-linear microstructural problems, such analyses are quite rare in the literature.

Han and Dawson ${ }^{202}$ proposed a multi-scale approach in which both micro and macro scale are modelled with the finite element method. The macro-scale is used to describe the structural components while the micro-scale is used to describe crystal ag-

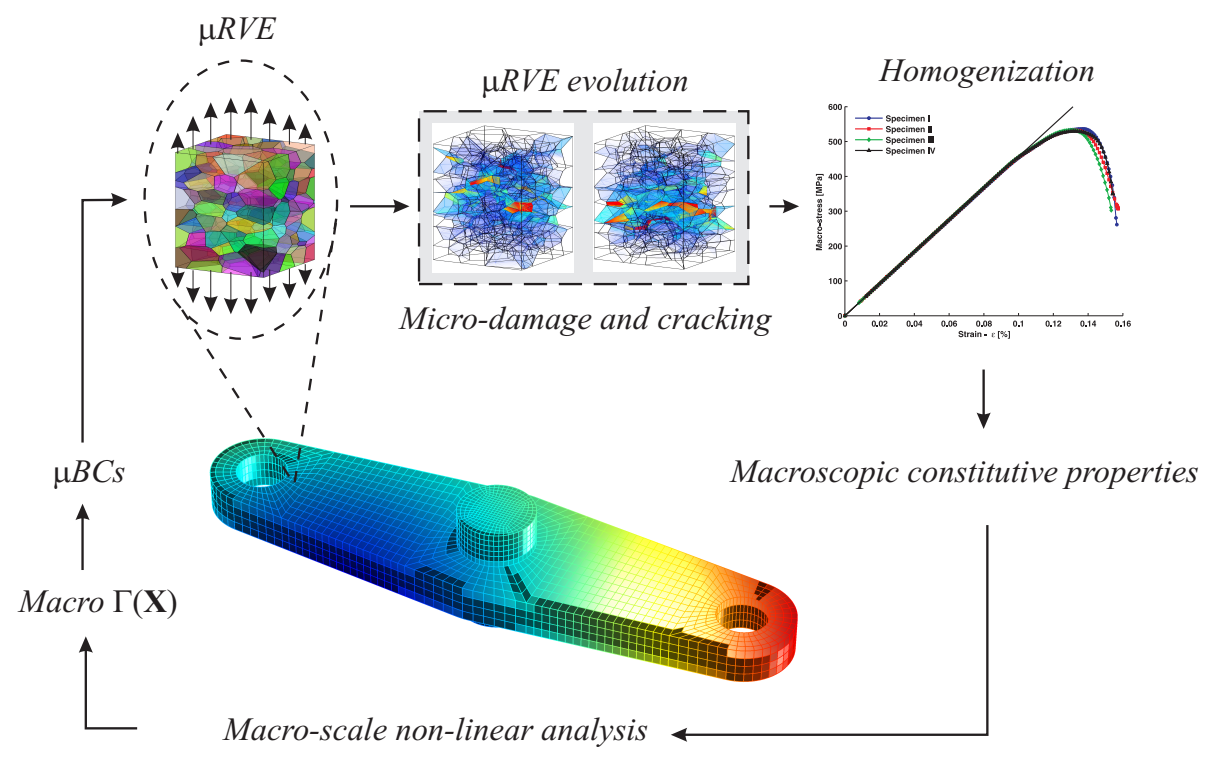

Fig. 4. Schematic of a homogenization based multiscale analysis: the macroscale provides boundary conditions for the micro-RVEs that evolve and provide local constitutive behavior to the macroscale. 
gregates. The individual crystals are represented as cubes and are spatially resolved using one or more finite elements. A crystal plasticity formulation is employed to describe the behavior of the aggregate. The solution process is strongly-coupled: the information needed at each scale is computed and exchanged concurrently throughout a simulation. In the localization process, the velocity gradient at a gauss point at a macro-scale mesh is assigned to a microscale mesh boundary in the form of equivalent essential (velocity) boundary conditions. The macroscale provides the velocity gradient at the macro Gauss points, which is projected onto the microscale meshes as essential boundary conditions (localization). The microscale provides the macro-stress and stiffness at the macro-points associated with the analyzed microstructures: to achieve this, an averaging procedure is required to determine the collective response of the aggregate (homogenization). The methodology has been successfully demonstrated on two three-dimensional applications.

Nakamachi et al. ${ }^{122}$ developed multi-scale finite element analysis procedures to evaluate macroscopic material properties, such as strength, yield loci and formability, by employing a realistic three dimensional (3D) micro polycrystal structure obtained by scanning electron microscopy and the electron backscattering diffraction (SEMEBSD) measurements. The polycrystalline homogenization algorithm was based on multi-scale asymptotic series expansion. The multi-scale finite element procedure involved discretization of both the macro continuum and the point attached micro crystal structure, which satisfies the periodicity condition. The method was applied to simulate the uniaxial tension test of a pure iron sheet to study the texture evolution at the micro level and subsequent yield loci at the macro level. Second, formability assessment tests for three automotive sheet metals were analyzed by a dynamic explicit FE code, to assess their formability. The thickness strain localization and the texture evolution were studied to characterize these sheet metals. The technique has been further developed for formability tests ${ }^{203}$ and accelerated with parallel computing techniques based on domain partitioning of $\mathrm{FE}$ model for macro-continuum using the message passing interface (MPI) library and PC clusters.

Rimoli and Ortiz ${ }^{204}$ proposed a three-dimensional model for intergranular hydrogen embrittlement taking into account the degradation of grain boundary strength arising from the grain-boundary diffusion of hydrogen. The microstructure was modelled using an idealized morphology and the intergranular interfaces were modelled with cohesive laws with strength dependent on the hydrogen concentration. The grains were modelled as anisotropic elasto-plastic domain deforming by crystallographic slip. The polycrystal was assumed to have random texture. The microstructural model was explicitly retained in the three-dimensional finite-element model of an AISI 4340 steel double-cantilever specimen in contact with an aggressive environment. The two scale were then computed concurrently: in particular, the full microstructure was represented in proximity of the crack tip, with a full crystal plasticity model; further from the crack tip, the microstructure was still represented, but only anisotropic elastic behavior was assumed; further from the process zone, 
the mesh was rapidly coarsened and the macro-material was assumed to be isotropic and elastic. The model was validated providing qualitative and quantitative predictions that matched well with experiments.

\section{General discussion and future developments}

Forty years after the first microstructure-based computations of polycrystals, twodimensional at that stage, presented by Canova et al. ${ }^{205}$, the CPFE approach has been adapted by many different teams all over the world: Cailletaud, Bohlke, Raabe, Gosh, Groeber, Dawson, Anand, ..., just to cite some of the renowned heading researchers. It has proved its great potential for providing an accurate constitutive modelling in cases of materials where the nature of the microstructure and of its heterogeneities play a major role in its mechanical response, not only polycrystalline materials but also e.g. ice ${ }^{58}$, carbon-filled rubber ${ }^{206}$, foams ${ }^{207} \ldots$ for which the principles of microstructure-based full field CPFEM have been extended.

As the approach consists in explicitly representing the microstructure (otherwise one may take advantage of significantly less time consuming mean-field approaches), numerical technical challenges had to faced: how to represent a high quality microstructure, how to mesh it correctly and how to have it computed with a compromise to be found between computing time/resources and the requirement of large size domains of computation ensuring statistical representativity. With these challenges, many studies have been presented in this review, which shows that efficient and robust solutions have been found. Yet developments are still necessary: polycrystalline microstructural morphologies can be much more complex than that provided by a classical Voronoi mosaic, because of non convexity of the grain morphologies, presence of different phases (as in multi-phase steels), morphological texture, multimodal grain size distribution ... Efforts are currently being made in this particular sense, notably in ${ }^{208}$. These efforts aim either at improving microstructure generation algorithms for introducing new features: multi-level tessellations meant to represent the multi-scale space subdivision of e.g. bainitic steels constituted of grains, packets, lamellae; processes of repulsion, weighting and spatio-temporal distribution of the tessellation nuclei enabling the generation of a wide variety of grain size distributions, including multi-modal. Besides, these efforts aim at developping a robust methodology for constructing a high quality mesh from experimental data: if experimental techniques are now able to provide a three-dimensional micron-scale representation of an actual polycrystalline microstructure, having this microstructure reproduced by a mesh is mainly performed with the basic method where to 1 voxel corresponds a brick element; this leads to step-wise representation of boundaries and interfaces which, as shown in many studies, can be at the origin of significant numerical artifacts in mechanical fields (especially stresses) and as a consequence to biased predictions of lifetime, probabilities of failure, ... Having an experimentally characterized microstructure processed to an unstructured mesh with grain bound- 
aries conforming to tetrahedral element boundaries is a very difficult task because of all the preliminary filtering steps to perform before, first, describing correctly the topology and, secondly, construct a high quality mesh from it.

The second aspect of the CPFEM on which major developments have been and will continue to be performed on a long run concerns the constitutive modelling accounting for microscopic mechanisms on a physics basis: dislocations motions, multiplication or annihilation, moving interfaces, diffusion processes, cleavage micro-cracks, void growth, coalescence, corrosion, ... Physics- or thermodynamics-based models exist and have proved their relevance for reproducing these mechanisms but the main difficulty is to have these models coupled to a FE resolution on a large scale $3 \mathrm{D}$ polycrystal. The compatibility can be ensured on a theoretical basis, as provided for example by the mechanics of generalized continua (see e.g. ${ }^{209}$ and an application in ${ }^{210}$ ), or on a small time and space scale of a physical process. This is the case for example of phase field modelling incorporating crystal plasticity ${ }^{211,212}$. But having phase field coupled to crystal plasticity, would it be by FE or not, in order to achieve a comprehensive description of deformation combined to phase transformation or recrystallisation involved in the process of elaboration of a metal alloy RVE remains a very high challenge. Once again, in this respect, synthetic microstructural representation still offers a good compromise, especially if one has to face with a system or a problem which is very demanding on the side of statistical representativity. This alternative solution, however, also has to deal with an ultimate difficulty arising as soon as there are modifications in the microstructure: how to have the mesh accounting for these modifications, would they be related to a change in material properties or to cracking, cavitation, ... The simplest way is to keep the same mesh and have the properties of the concerned elements varying such as to reproduce the consequences of the material properties variations. This consists for example in dropping the stiffness of an element to a quasi null value in order to mimic the presence of a crack. This solution however cannot properly apply to crack propagation since its velocity is strongly dependent on the mesh refinement at the tip of the crack. One thus has to resort to mesh reconstruction and field transfer from increment to increment. Combined to non-linearities and anisotropies (which are typical of crystal plasticity), significant problems of numerical convergence arise. This kind of problem summarizes most of the challenges in CPFEM: multi-scale and multi-physics problems engaging complex numerical methods for the microstructure generation, the meshing-remeshing, numerical resolution and comprehensive physics-based constitutive modelling.

\section{Conclusions}

In the present overview, recent contributions on three-dimensional mechanical modelling of polycrystalline materials at the grain-scale have been surveyed. The computational analysis of fully 3D microstructures, with accurate representation of the micro-morphology, has recently gained momentum, due to wider affordability of 
High Performance Computing. Several aspects, ranging from the generation and meshing of more or less accurate microstructures, to the use of more or less refined constitutive models for the individual grains, to the formulation of multiscale models involving the component and the grain scales, have been considered and recent contributions have been discussed. There is today wide acceptance that three dimensional aspects (morphology and constitutive laws) have to be considered in the analysis, if the full complexity of local microstructural phenomena and rare events, affecting especially the behavior of the material with respect to damage and failures, has to be fully understood. On the other hand, some limitations of continuum approaches for the study of some phenomena have been pointed out in some studies, calling for the use of physically based models involving scales below the grain level.

\section{Acknowledgments}

This research was partially supported by a Marie Curie Intra European Fellowship within the 7th European Community Framework Programme (Project No 274161).

\section{References}

1. Z. Hashin, "Analysis of Composite Materials - A survey," ASME Journal of Applied Mechanics, vol. 50, pp. 481-505, 1983.

2. T. Mura, Micromechanics of defects in solids. Mechanics of Elastic and Inelastic Solids, Dordrecht, The Netherlands: Kluwer Academic Publishers, second revised edition ed., 1987.

3. S. Nemat-Nasser and M. Hori, Micromechanics: overall properties of heterogeneous materials. Amsterdam, The Netherlands: North-Holland, Elsevier, second revised edition ed., 1999.

4. A. Needleman, "Computational mechanics at the mesoscale," Acta Materialia, vol. 48, no. 1 , pp. $105-124,2000$.

5. T. Watanabe and S. Tsurekawa, "The control of brittleness and development of desirable mechanical properties in polycrystalline systems by grain boundary engineering," Acta Materialia, vol. 47, pp. 4171 - 4185, 1999.

6. B. Adams and T. Olson, "The mesostructure - prperties linkage in polycrystals," Progress in Materials Science, vol. 43, pp. 1-88, 1998.

7. K. Döbrich, C. Rau, and C. Krill III, "Quantitative characterization of the threedimensional microstructure of polycrystalline Al-Sn using X-ray microtomography," Metallurgical and Materials Transactions A, vol. 35A, pp. 1953-1961, 2004.

8. C. Zhang, A. Suzuki, T. Ishimaru, and M. Enomoto, "Characterization of Threedimensional grain structure in polycrystalline iron by serial sectioning," Metallurgical and Materials Transactions A, vol. 35A, pp. 1927-1933, 2004.

9. M. Groeber, B. Haley, M. Uchic, D. Dimiduk, and S. Gosh, "3D reconstruction and characterization of polycrystalline microstructures using a FIB-SEM system," Materials Characterization, pp. 259-273.

10. A. King, G. Johnson, D. Engelberg, W. Ludwig, and J. Marrow, "Observations of intergranular stress corrosion cracking in a grain-mapped polycrystal," Science, vol. 321, no. 5887, pp. 382-385, 2008.

11. M. Groeber, S. Ghosh, M. Uchic, and D. Dimiduk, "A framework for automated analysis and simulation of 3D polycrystalline microstructures. Part 1: Statistical characterization," Acta Materialia, vol. 56, pp. 1257-1273, 2008. 
12. W. Ludwig, A. King, P. Reischig, M. Herbig, E. Lauridsen, S. Schmidt, H. Proudhon, S. Forest, P. Cloetens, S. R. du Roscoat, J. Buffire, T. Marrow, and H. Poulsen, "New opportunities for $3 \mathrm{D}$ materials science of polycrystalline materials at the micrometre lengthscale by combined use of X-ray diffraction and X-ray imaging," Materials Science and Engineering: A, vol. 524, pp. $69-76,2009$.

13. S. Rahimi, D. Engelberg, J. Duff, and T. Marrow, "In situ observation of intergranular crack nucleation in a grain boundary controlled austenitic stainless steel," Journal of Microscopy, vol. 233, no. 3, pp. 423-431, 2009.

14. M. Herbig, A. King, P. Reischig, H. Proudhon, E. Lauridsen, J. Marrow, J.-Y. Buffire, and W. Ludwig, "3-D growth of a short fatigue crack within a polycrystalline microstructure studied using combined diffraction and phase-contrast X-ray tomography," Acta Materialia, vol. 59, no. 2, pp. 590 - 601, 2011.

15. A. King, W. Ludwig, M. Herbig, J.-Y. Buffire, A. Khan, N. Stevens, and T. Marrow, "Three-dimensional in situ observations of short fatigue crack growth in magnesium," Acta Materialia, vol. 59, no. 17, pp. 6761 - 6771, 2011.

16. T. Zohdi and P. Wriggers, An introduction to computational micromechanics, vol. 20 of Lecture Notes in Applied and Computational Mechanics. Berlin: Springer, 2005.

17. F. Roters, P. Eisenlohr, L. Hantcherli, D. Tjahjanto, T. Bieler, and D. Raabe, "Overview of constitutive laws, kinematics, homogenization and multiscale methods in crystal plasticity finite-element modeling: Theory, experiments, applications," Acta Materialia, vol. 58, no. 4, pp. 1152 - 1211, 2010.

18. I. Simonovski and L. Cizelj, "Automatic parallel generation of finite element meshes for complex spatial structures," Computational Materials Science, vol. 50, no. 5, pp. $1606-1618,2011$.

19. I. Simonovski and L. Cizelj, "Computational multiscale modeling of intergranular cracking," Journal of Nuclear Materials, vol. 414, no. 2, pp. 243 - 250, 2011.

20. I. Simonovski and L. Cizelj, "Towards modeling intergranular stress corrosion cracks on grain size scales," Nuclear Engineering and Design, vol. 246, no. 0, pp. 107 - 114, 2012 .

21. J. A. R. Bomidi, N. Weinzapfel, and F. Sadeghi, "Three-dimensional modelling of intergranular fatigue failure of fine grain polycrystalline metallic mems devices," Fatigue \& Fracture of Engineering Materials \& Structures, pp. no-no, 2012.

22. A. Crocker, P. Flewitt, and S. G.E., "Computational modelling of fracture in polycrystalline materials," International Materials Reviews, vol. 50, no. 2, pp. 99-124, 2005.

23. G. Smith, A. Crocker, G. Hughes, and P. Flewitt, "Three-dimensional modelling of fracture in polycrystals," in Fracture of Nano and Engineering Materials and Structures (E. Gdoutos, ed.), pp. 823-824, Springer Netherlands, 2006.

24. T. Marrow, L. Babout, A. Jivkov, P. Wood, D. Engelberg, N. Stevens, P. Withers, and R. Newman, "Three dimensional observations and modelling of intergranular stress corrosion cracking in austenitic stainless steel," Journal of Nuclear Materials, vol. 352 , pp. $62-74,2006$.

25. G. Hughes, G. Smith, P. Flewitt, and A. Crocker, "The brittle fracture of polycrystalline zinc," Proceedings of the Royal Society A: Mathematical, Physical and Engineering Science, vol. 463, no. 2085, pp. 2129-2151, 2007.

26. D. Catoor and K. Kumar, "Crackgrain boundary interactions in zinc bicrystals," Philosophical Magazine, vol. 91, no. 16, pp. 2154-2185, 2011.

27. A. Zeghadi, F. N'guyen, S. Forest, A.-F. Gourgues, and O. Bouaziz, "Ensemble averaging stress-strain fields in polycrystalline aggregates with a constrained surface microstructure - part 1: anisotropic elastic behaviour," Philosophical Magazine, vol. 87, 
no. 8-9, pp. 1401-1424, 2007.

28. A. Zeghadi, S. Forest, A.-F. Gourgues, and O. Bouaziz, "Ensemble averaging stressstrain fields in polycrystalline aggregates with a constrained surface microstructure - part 2: crystal plasticity," Philosophical Magazine, vol. 87, no. 8-9, pp. 1425-1446, 2007.

29. G. Cailletaud, S. Forest, D. Jeulin, F. Feyel, I. Galliet, V. Mounoury, and S. Quilici, "Some elements of microstructural mechanics," Computational Materials Science, vol. 27 , no. 3 , pp. $351-374,2003$.

30. I. Simonovski and L. Cizelj, "Grain-scale modeling approaches for polycrystalline aggregates," in Polycrystalline Materials - Theoretical and Practical Aspects (Z. Zakhariev, ed.), vol. 125(1), pp. 49-74, Oxford: InTech, 2012.

31. R. Quey, P. Dawson, and F. Barbe, "Large scale 3D random polycrystals for the finite element method: Generation, meshing and remeshing," Computer Methods in Applied Mechanics and Engineering, vol. 200, pp. 1729-1745, 2011.

32. C. N'Guyen, F. Barbe, N. Osipov, G. Cailletaud, B. Marini, and C. Petry, "Micromechanical local approach to brittle failure in bainite high resolution polycrystals: A short presentation," Computational Materials Science, vol. 64, pp. 62-65, 2012.

33. S. I. Ranganathan and M. Ostoja-Starzewski, "Scaling function, anisotropy and the size of RVE in elastic random polycrystals," Journal of the Mechanics and Physics of Solids, vol. 56, no. 9, pp. 2773 - 2791, 2008.

34. H. Ritz and P. Dawson, "Sensitivity to grain discretization of the simulated crystal stress distributions in FCC polycrystals," Modelling and Simulation in Materials Science and Engineering, vol. 17, no. 1, p. 015001, 2009.

35. D. Raabe, Z. Zhao, and W. Mao, "On the dependence of in-grain subdivision and deformation texture of aluminum on grain interaction," Acta Materialia, vol. 50, no. 17 , pp. $4379-4394,2002$.

36. Z. Zhao, S. Kuchnicki, R. Radovitzky, and A. Cuitiño, "Influence of in-grain mesh resolution on the prediction of deformation textures in fcc polycrystals by crystal plasticity FEM," Acta Materialia, vol. 55, no. 7, pp. 2361 - 2373, 2007.

37. S. Kalidindi, C. Bronkhorst, and L. Anand, "Crystallographic texture evolution in bulk deformation processing of FCC metals," Journal of the Mechanics and Physics of Solids, vol. 40, no. 3, pp. 537 - 569, 1992.

38. A. Beaudoin, P. Dawson, K. Mathur, and U. Kocks, "A hybrid finite element formulation for polycrystal plasticity with consideration of macrostructural and microstructural linking," International Journal of Plasticity, vol. 11, no. 5, pp. 501 $521,1995$.

39. G. Sarma and P. Dawson, "Effects of interactions among crystals on the inhomogeneous deformations of polycrystals," Acta Materialia, vol. 44, no. 5, pp. 1937 - 1953, 1996.

40. G. Sarma and D. P.R., "Texture predictions using a polycrystal plasticity model incorporating neighbor interactions," International Journal of Plasticity, vol. 12, no. 8, pp. $1023-1054,1996$.

41. A. J. Beaudoin, H. Mecking, and U. F. Kocks, "Development of localized orientation gradients in fcc polycrystals," Philosophical Magazine A, vol. 73, no. 6, pp. 1503$1517,1996$.

42. V. Bachu and S. R. Kalidindi, "On the accuracy of the predictions of texture evolution by the finite element technique for fcc polycrystals," Materials Science and Engineering: A, vol. 257, no. 1, pp. 108 - 117, 1998. International Conference on Crystallographic Texture.

43. C. A. Bronkhorst, S. R. Kalidindi, and L. Anand, "Polycrystalline plasticity and the 
evolution of crystallographic texture in FCC metals," Philosophical Transactions of the Royal Society of London. Series A: Physical and Engineering Sciences, vol. 341, no. 1662 , pp. $443-477,1992$.

44. P. V. Houtte, L. Delannay, and S. Kalidindi, "Comparison of two grain interaction models for polycrystal plasticity and deformation texture prediction," International Journal of Plasticity, vol. 18, no. 3, pp. 359 - 377, 2002.

45. D. P. Mika and P. R. Dawson, "Effects of grain interaction on deformation in polycrystals," Materials Science and Engineering: A, vol. 257, no. 1, pp. 62 - 76, 1998. International Conference on Crystallographic Texture.

46. D. Mika and P. Dawson, "Polycrystal plasticity modeling of intracrystalline boundary textures," Acta Materialia, vol. 47, no. 4, pp. 1355 - 1369, 1999.

47. P. Bate and J. Q. da Fonseca, "Texture development in the cold rolling of IF steel," Materials Science and Engineering: A, vol. 380, no. 12, pp. 365 - 377, 2004.

48. A. Jivkov, N. Stevens, and T. Marrow, "A three-dimensional computational model for intergranular cracking," Computational Materials Science, vol. 38, no. 2, pp. 442 $-453,2006$.

49. L. Delannay, P. J. Jacques, and S. R. Kalidindi, "Finite element modeling of crystal plasticity with grains shaped as truncated octahedrons," International Journal of Plasticity, vol. 22, no. 10, pp. 1879 - 1898, 2006.

50. S. Kumar and S. Kurtz, "Simulation of material microstructure using a 3D Voronoi tessellation: Calculation of effective thermal expansion coefficient of polycrystalline materials," Acta Metallica, vol. 42, pp. 3917-3927, 1994.

51. S. Kumar, S. Kurtz, and V. Agarwala, "Micro-stress distribution within polycrystalline aggregate," Acta Mechanica, vol. 114, pp. 203-216, 1996.

52. K. J. Kozaczek, B. G. Petrovic, C. O. Ruud, S. K. Kurtz, and A. R. McIlree, "Microstructural modelling of grain-boundary stresses in alloy 600," Journal of Materials Science, vol. 30, pp. 2390-2400, 1995.

53. F. Barbe, L. Decker, D. Jeulin, and G. Cailletaud, "Intergranular and intragranular behavior of polycrystalline aggregates. Part 1: F.E. model," International Journal of Plasticity, vol. 17, pp. 513-536, 2001.

54. F. Barbe, S. Forest, and G. Cailletaud, "Intergranular and intragranular behavior of polycrystalline aggregates. Part 2: Results," International Journal of Plasticity, vol. 17 , pp. 537-563, 2001.

55. F. Barbe and R. Quey, "A numerical modelling of 3D polycrystal-to-polycrystal diffusive phase transformations involving crystal plasticity," International Journal of Plasticity, vol. 27, no. 6, pp. 823 - 840, 2011.

56. M. Nygårds and P. Gudmundson, "Three-dimensional periodic Voronoi grain models and micromechanical FE-simulations of a two phase steel," Computational Material Science, vol. 24, pp. 513-519, 2002.

57. M. Nygårds, "Number of grains necessary to homogenize elastic materials with cubic symmetry," Mechanics of Materials, vol. 35, pp. 1049-1057, 2003.

58. T. Kanit, S. Forest, I. Galliet, V. Mounoury, and D. Jeulin, "Determination of the size of the representative volume element for random composites: statistical and numerical approach," International Journal of Solids and Structures, vol. 40, no. 1314, pp. $3647-3679,2003$.

59. Y. Zhao and R. Tyron, "Automatic 3-D simulation and micro-stress distribution of polycrystalline metallic materials," Computer Methods in Applied Mechanics and Engineering, vol. 193, pp. 3919-3934, 2004.

60. O. Diard, S. Leclercq, G. Rousselier, and G. Cailletaud, "Evaluation of finite element based analysis of 3D multicrystalline aggregates plasticity: Application to crystal 
plasticity model identification and the study of stress and strain fields near grain boundaries," International Journal of Plasticity, vol. 21, no. 4, pp. 691 - 722, 2005.

61. K. Zhang, M. Wu, and R. Feng, "Simulation of microplasticity-induced deformation in uniaxially strained ceramics by $3-\mathrm{D}$ voronoi polycrystal modeling," International Journal of Plasticity, vol. 21, no. 4, pp. $801-834,2005$.

62. M. Kamaya, Y. Kawamura, and T. Kitamura, "Three-dimensional local stress analysis on grain boundaries in polycrystalline material," International Journal of Solids Es Structures, vol. 44, pp. 3267-3277, 2007.

63. N. Osipov, A.-F. Gourgues-Lorenzon, B. Marini, V. Mounoury, F. Nguyen, and G. Cailletaud, "Fe modelling of bainitic steels using crystal plasticity," Philosophical Magazine, vol. 88, no. 30-32, pp. 3757-3777, 2008.

64. J. Mathieu, K. Inal, S. Berveiller, and O. Diard, "A micromechanical interpretation of the temperature dependence of Beremin model parameters for french RPV steel," Journal of Nuclear Materials, vol. 406, pp. 97-112, 2010.

65. M. Libert, C. Rey, L. Vincent, and B. Marini, "Temperature dependant polycrystal model application to bainitic steel behavior under tri-axial loading in the ductilebrittle transition," International Journal of Solids and Structures, vol. 48, pp. 21962208, 2011.

66. T. Luther and C. Könke, "Polycrystal models for the analysis of intergranular crack growth in metallic materials," Engineering Fracture Mechanics, vol. 76, no. 15, pp. $2332-2343,2009$.

67. A. Musienko and G. Cailletaud, "Simulation of inter- and transgranular crack propagation in polycrystalline aggregates due to stress corrosion cracking," Acta Materialia, vol. 57, no. 13 , pp. $3840-3855,2009$.

68. N. Weinzapfel, F. Sadeghi, and V. Bakolas, "An approach for modeling material grain structure in investigations of hertzian subsurface stresses and rolling contact fatigue," Journal of Tribology, vol. 132, no. 4, 2010.

69. I. Benedetti and M. Aliabadi, "A three-dimensional grain boundary formulation for microstructural modelling of polycrystalline materials," Computational Material Science, 2012.

70. I. Benedetti and M. Aliabadi, "A three-dimensional cohesive-frictional grainboundary micromechanical model for intergranular degradation and failure in polycrystalline materials," Computer Methods in Applied Mechanics and Engineering, vol. 265, no. 0, pp. $36-62,2013$.

71. M. G. Mecozzi, J. Sietsma, and S. van der Swaag, "Analysis of $\gamma \longrightarrow \alpha$ transformation in a Nb micro-alloyed C-Mn steel by phase field modelling," Acta Materialia, vol. 54, pp. 1431-1440, 2006.

72. M. Bernacki, Y. Chastel, T. Coupez, and R. Log, "Level set framework for the numerical modelling of primary recrystallization in polycrystalline materials," Scripta Materialia, vol. 58, pp. 1129-1132, 2008.

73. M. Apel, S. Benke, and I. Steinbach, "Virtual dilatometer curves and effective young's modulus of a 3D multiphase structure calculated by the phase-field method," Computational Materials Science, vol. 45, pp. 589-592, 2009.

74. A. Malik, H. K. Yeddu, G. Amberg, A. Borgenstam, and J. Agren, "Three dimensional elasto-plastic phase field simulation of martensitic transformation in polycrystal," Materials Science and Engineering A, vol. 556, pp. 221-232, 2012.

75. S. Kumar, S. Kurtz, J. Banavar, and M. Sharma, "Properties of a three-dimensional Poisson-Voronoi tessellation: a Monte Carlo study," Journal of Statistical Physics, vol. 67 , no. 3/4, pp. 523-551, 1992.

76. S. Kumar and S. Kurtz, "A Monte Carlo study of size and angular properties of 
a three-dimensional Poisson-Delaunay cell," Journal of Statistical Physics, vol. 75, no. $3 / 4$, pp. $735-748,1994$.

77. S. Kumar and S. Kurtz, "Monte-Carlo study of angular and edge length distributions in a three-dimensional Poisson-Voronoi tessellation," Materials Characterization, vol. 34, pp. 15-27, 1995.

78. M. Tanemura, "Statistical distributuions of Poisson Voronoi cells in two and three dimensions," Forma, vol. 18, pp. 221-247, 2003.

79. F. Fritzen, T. Böhlke, and E. Schnack, "Periodic three-dimensional mesh generation for crystalline aggregates based on Voronoi tessellations," Computational Mechanics, vol. 43, pp. 701-713, 2009.

80. Z. Fan, Y. Wu, X. Zhao, and Y. Lu, "Simulation of polycrystalline structure with voronoi diagram in laguerre geometry based on random closed packing of spheres," Computational Materials Science, vol. 29, no. 3, pp. 301 - 308, 2004.

81. N. Nouri, V. Ziaei-Rad, and S. Ziaei-Rad, "An approach for simulating microstructures of polycrystalline materials," Computational Mechanics, vol. 52, no. 1, pp. 181192, 2013.

82. F. Lavergne, R. Brenner, and K. Sab, "Effects of grain size distribution and stress heterogeneity on yield stress of polycrystals: A numerical approach," Computational Materials Science, vol. 77, no. 0, pp. 387 - 398, 2013.

83. C. Rycroft, "Voro++: A three-dimensional Voronoi cell library in $\mathrm{C}++$, , Chaos, vol. 19, p. 041111, 2009.

84. C. B. Barber, D. P. Dobkin, and H. Huhdanpaa, "The quickhull algorithm for convex hulls," ACM Trans. Math. Softw., vol. 22, pp. 469-483, Dec. 1996.

85. M. A. Miodownik, "A review of microstructural computer models used to simulate grain growth and recrystallisation in aluminium alloys," Journal of Light Metals, vol. 2, no. 3, pp. 125 - 135, 2002. Modelling of Light Metals.

86. M. P. Anderson, G. S. Grest, and D. J. Srolovitz, "Computer simulation of normal grain growth in three dimensions," Philosophical Magazine Part B, vol. 59, no. 3, pp. 293-329, 1989.

87. J. A. Glazier, "Grain growth in three dimensions depends on grain topology," Phys. Rev. Lett., vol. 70, pp. 2170-2173, Apr 1993.

88. Y. Saito, "Monte carlo simulation of grain growth in three-dimensions," ISIJ International, vol. 38, no. 6, pp. 559-566, 1998.

89. A. Miyake, "Monte carlo simulation of normal grain growth in 2- and 3-dimensions: the lattice-model-independent grain size distribution," Contributions to Mineralogy and Petrology, vol. 130, no. 2, pp. 121-133, 1998.

90. Y. Kim, S. Hwang, M. Kim, S. Kwun, and S. Chae, "Three-dimensional montecarlo simulation of grain growth using triangular lattice," Materials Science and Engineering: A, vol. 408, no. 12, pp. $110-120,2005$.

91. G. Sarma, B. Radhakrishnan, and T. Zacharia, "Finite element simulations of cold deformation at the mesoscale," Computational Materials Science, vol. 12, no. 2, pp. $105-123,1998$.

92. F. Wakai, N. Enomoto, and H. Ogawa, "Three-dimensional microstructural evolution in ideal grain growth - general statistics," Acta Materialia, vol. 48, no. 6, pp. 1297 1311, 2000.

93. F. Wakai, Y. Shinoda, S. Ishihara, and A. Domnguez-Rodrguez, "Topological transformation of grains in three-dimensional normal grain growth," Journal of Materials Research, vol. 16, pp. 2136-2142, 72001.

94. E. A. Lazar, J. K. Mason, R. D. MacPherson, and D. J. Srolovitz, "A more accurate three-dimensional grain growth algorithm," Acta Materialia, vol. 59, no. 17, pp. 6837 
$-6847,2011$

95. K. Fuchizaki, T. Kusaba, and K. Kawasaki, "Computer modelling of threedimensional cellular pattern growth," Philosophical Magazine Part B, vol. 71, no. 3, pp. 333-357, 1995.

96. D. Weygand, Y. Brchet, J. Lpinoux, and W. Gust, "Three-dimensional grain growth: A vertex dynamics simulation," Philosophical Magazine Part B, vol. 79, no. 5, pp. 703-716, 1999.

97. M. Syha and D. Weygand, "A generalized vertex dynamics model for grain growth in three dimensions," Modelling and Simulation in Materials Science and Engineering, vol. 18, no. 1, p. 015010, 2010.

98. H. Ding, Y. He, L. Liu, and W. Ding, "Cellular automata simulation of grain growth in three dimensions based on the lowest-energy principle," Journal of Crystal Growth, vol. 293, no. 2, pp. $489-497,2006$.

99. C. K. III and L.-Q. Chen, "Computer simulation of 3-D grain growth using a phasefield model," Acta Materialia, vol. 50, no. 12, pp. 3059 - 3075, 2002.

100. Y. Suwa, Y. Saito, and H. Onodera, "Phase field simulation of grain growth in three dimensional system containing finely dispersed second-phase particles," Scripta Materialia, vol. 55, no. 4, pp. 407 - 410, 2006.

101. Y. Suwa, Y. Saito, and H. Onodera, "Three-dimensional phase field simulation of the effect of anisotropy in grain-boundary mobility on growth kinetics and morphology of grain structure," Computational Materials Science, vol. 40, no. 1, pp. 40 - 50, 2007.

102. Y. Suwa, Y. Saito, and H. Onodera, "Parallel computer simulation of threedimensional grain growth using the multi-phase-field model," Materials Transactions, vol. 49, no. 4, pp. 704-709, 2008.

103. L. Vanherpe, N. Moelans, B. Blanpain, and S. Vandewalle, "Bounding box algorithm for three-dimensional phase-field simulations of microstructural evolution in polycrystalline materials," Phys. Rev. E, vol. 76, p. 056702, Nov 2007.

104. N. Moelans, B. Blanpain, and P. Wollants, "An introduction to phase-field modeling of microstructure evolution," Calphad, vol. 32, no. 2, pp. 268 - 294, 2008.

105. I. Singer-Loginova and H. M. Singer, "The phase field technique for modeling multiphase materials," Reports on Progress in Physics, vol. 71, no. 10, p. 106501, 2008.

106. D. Rowenhorst, A. Lewis, and G. Spanos, "Three-dimensional analysis of grain topology and interface curvature in a $\beta$-titanium alloy," Acta Materialia, vol. 58, pp. 55115519, 2010.

107. J. Spowart, H. Mullens, and B. Puchala, "Collecting and analyzing microstructures in three dimensions: A fully automated approach," JOM, vol. 55, no. 10, pp. 35-37, 2003.

108. J. E. Spowart, "Automated serial sectioning for 3-D analysis of microstructures," Scripta Materialia, vol. 55, no. 1, pp. 5-10, 2006.

109. A. Ullah, G. Liu, H. Wang, M. Khan, D. F. Khan, and J. Luan, "Optimal approach of three-dimensional microstructure reconstructions and visualizations," Materials Express, vol. 3, no. 2, pp. 109-118, 2013.

110. A. Lewis, J. Bingert, D. Rowenhorst, A. Gupta, A. Geltmacher, and G. Spanos, "Two- and three-dimensional microstructural characterization of a super-austenitic stainless steel," Materials Science and Engineering: A, vol. 418, no. 1-2, pp. 11-18, 2006.

111. D. Rowenhorst, A. Gupta, C. Feng, and G. Spanos, "3D crystallographic and morphological analysis of coarse martensite: Combining EBSD and serial sectioning," Scripta Materialia, vol. 55, no. 1, pp. 11 - 16, 2006. 
112. M. D. Uchic, M. A. Groeber, D. M. Dimiduk, and J. Simmons, "3D microstructural characterization of nickel superalloys via serial-sectioning using a dual beam FIBSEM," Scripta Materialia, vol. 55, no. 1, pp. 23 - 28, 2006.

113. M. Groeber, S. Ghosh, M. Uchic, and D. Dimiduk, "A framework for automated analysis and simulation of $3 \mathrm{D}$ polycrystalline microstructures. Part 2: Synthetic structure generation," Acta Materialia, vol. 56, pp. 1274-1287, 2008.

114. S. Zaefferer, S. Wright, and D. Raabe, "Three-dimensional orientation microscopy in a focused ion beam-scanning electron microscope: A new dimension of microstructure characterization," Metallurgical and Materials Transactions A, vol. 39, no. 2, pp. 374389, 2008.

115. R. Wirth, "Focused ion beam (FIB) combined with SEM and TEM: Advanced analytical tools for studies of chemical composition, microstructure and crystal structure in geomaterials on a nanometre scale," Chemical Geology, vol. 261, no. 34, pp. 217 229, 2009.

116. P. Munroe, "The application of focused ion beam microscopy in the material sciences," Materials Characterization, vol. 60, no. 1, pp. 2 - 13, 2009.

117. T. Sumigawa, T. Kitamura, and K. Ohishi, "Slip behaviour near a grain boundary in high-cycle fatigue of poly-crystal copper," Fatigue $\&$ Fracture of Engineering Materials \& Structures, vol. 27, no. 6, pp. 495-503, 2004.

118. A. Lewis and A. Geltmacher, "Image-based modeling of the response of experimental 3D microstructures to mechanical loading," Scripta Materialia, vol. 55, no. 1, pp. 81 $-85,2006$.

119. Y. Bhandari, S. Sarkar, M. Groeber, M. Uchic, D. Dimiduk, and S. Ghosh, "3D polycrystalline microstructure reconstruction from FIB generated serial sections for FE analysis," Computational Materials Science, vol. 41, no. 2, pp. 222 - 235, 2007.

120. A. Musienko, A. Tatschl, K. Schmidegg, O. Kolednik, R. Pippan, and G. Cailletaud, "Three-dimensional finite element simulation of a polycrystalline copper specimen," Acta Materialia, vol. 55, pp. 4121-4136, 2007.

121. M. S. Qidwai, A. C. Lewis, and A. B. Geltmacher, "Using image-based computational modeling to study microstructure-yield correlations in metals," Acta Materialia, vol. 57, no. 14 , pp. $4233-4247,2009$.

122. E. Nakamachi, N. Tam, and H. Morimoto, "Multi-scale finite element analyses of sheet metals by using SEM-EBSD measured crystallographic RVE models," International Journal of Plasticity, vol. 23, no. 3, pp. 450-489, 2007.

123. A. Bhattacharyya, E. El-Danaf, S. R. Kalidindi, and R. D. Doherty, "Evolution of grain-scale microstructure during large strain simple compression of polycrystalline aluminum with quasi-columnar grains: OIM measurements and numerical simulations," International Journal of Plasticity, vol. 17, no. 6, pp. 861 - 883, 2001.

124. X. You, T. Connolley, P. McHugh, H. Cuddy, and C. Motz, "A combined experimental and computational study of deformation in grains of biomedical grade 316lvm stainless steel," Acta Materialia, vol. 54, no. 18, pp. 4825 - 4840, 2006.

125. A. Brahme, M. Alvi, D. Saylor, J. Fridy, and A. Rollett, "3D reconstruction of microstructure in a commercial purity aluminum," Scripta Materialia, vol. 55, no. 1, pp. $75-80,2006$.

126. L. St-Pierre, E. Héripré, M. Dexet, J. Crépin, G. Bertolino, and N. Bilger, "3D simulations of microstructure and comparison with experimental microstructure coming from O.I.M analysis," International Journal of Plasticity, vol. 24, no. 9, pp. 1516 $1532,2008$.

127. C. Gérard, F. N'Guyen, N. Osipov, G. Cailletaud, M. Bornert, and D. Caldemaison, "Comparison of experimental results and finite element simulation of strain 
localization scheme under cyclic loading," Computational Materials Science, vol. 46, pp. $755-760,2009$.

128. S. Stock, "X-ray microtomography of materials," International Materials Reviews, vol. 44, no. 4, pp. 141-164, 1999.

129. U. Bonse and F. Busch, "X-ray computed microtomography ( $\mu \mathrm{CT})$ using synchrotron radiation (SR)," Progress in Biophysics and Molecular Biology, vol. 65, no. 12, pp. 133 - 169, 1996.

130. W. Ludwig and D. Bellet, "Penetration of liquid gallium into the grain boundaries of aluminium: a synchrotron radiation microtomographic investigation," Materials Science and Engineering: A, vol. 281, no. 12, pp. 198 - 203, 2000.

131. E. Maire, J. Y. Buffire, L. Salvo, J. J. Blandin, W. Ludwig, and J. M. Ltang, "On the application of X-ray microtomography in the field of materials science," Advanced Engineering Materials, vol. 3, no. 8, pp. 539-546, 2001.

132. S. Nielsen, E. Lauridsen, D. J. Jensen, and H. Poulsen, "A three-dimensional X-ray diffraction microscope for deformation studies of polycrystals," Materials Science and Engineering: A, vol. 319-321, no. 0, pp. 179 - 181, 2001.

133. B. Larson, W. Yang, G. Ice, J. Budai, and J. Tischler, "Three-dimensional X-ray structural microscopy with submicrometre resolution," Nature, vol. 415, no. 6874, pp. 887-890, 2002.

134. W. Ludwig, J.-Y. Buffire, S. Savelli, and P. Cloetens, "Study of the interaction of a short fatigue crack with grain boundaries in a cast Al alloy using X-ray microtomography," Acta Materialia, vol. 51, no. 3, pp. 585 - 598, 2003.

135. H. F. Poulsen, S. F. Nielsen, E. M. Lauridsen, S. Schmidt, R. M. Suter, U. Lienert, L. Margulies, T. Lorentzen, and D. Juul Jensen, "Three-dimensional maps of grain boundaries and the stress state of individual grains in polycrystals and powders," Journal of Applied Crystallography, vol. 34, pp. 751-756, Dec 2001.

136. H. F. Poulsen, Three-dimensional X-ray diffraction microscopy: mapping polycrystals and their dynamics, vol. 205. Springer, 2004.

137. A. King, M. Herbig, W. Ludwig, P. Reischig, E. Lauridsen, T. Marrow, and J. Buffière, "Non-destructive analysis of micro texture and grain boundary character from X-ray diffraction contrast tomography," Nuclear Instruments and Methods in Physics Research Section B: Beam Interactions with Materials and Atoms, vol. 268, no. 3-4, pp. $291-296,2010$.

138. W. Ludwig, S. Schmidt, E. M. Lauridsen, and H. F. Poulsen, "X-ray diffraction contrast tomography: a novel technique for three-dimensional grain mapping of polycrystals. I. Direct beam case," Journal of Applied Crystallography, vol. 41, pp. 302-309, Apr 2008.

139. G. Johnson, A. King, M. G. Honnicke, J. Marrow, and W. Ludwig, "X-ray diffraction contrast tomography: a novel technique for three-dimensional grain mapping of polycrystals. II. The combined case," Journal of Applied Crystallography, vol. 41, pp. 310-318, Apr 2008.

140. T. Böhlke, K. Jöchen, O. Kraft, D. Löhe, and V. Schulze, "Elastic properties of polycrystalline microcomponents," Mechanics of Materials, vol. 42, no. 1, pp. 11 23, 2010.

141. F. Trentacoste, I. Benedetti, and M. H. Aliabadi, "Computational homogenization of polycrystalline materials with pores: a three-dimensional grain boundary formulation," Journal of Multiscale Modelling, vol. 04, no. 03, p. 1250012, 2012.

142. H. Resk, L. Delannay, M. Bernacki, T. Coupez, and R. Logé, "Adaptive mesh refinement and automatic remeshing in crystal plasticity finite element simulations," Modelling and Simulation in Materials Science and Engineering, vol. 17, no. 7, p. 075012, 
2009.

143. S. Ghosh, Y. Bhandari, and M. Groeber, "CAD-based reconstruction of 3D polycrystalline alloy microstructures from FIB generated serial sections," Computer-Aided Design, vol. 40, no. 3, pp. 293 - 310, 2008.

144. M. Mehrabadi and S. Cowin, "Eigentensors of linear anisotropic elastic materials," Quarterly Journal of Mechanics and Applied Mathematics, vol. 43, no. 1, pp. 15-41, 1990.

145. M. Ostoja-Starzewski, "Material spatial randomness: from statistical to representative volume element," Probabilistic Engineering Mechanics, vol. 21, pp. 112-132, 2006.

146. R. J. Asaro, "Micromechanics of crystals and polycrystals," Advances in applied mechanics, vol. 23, no. 1, 1983.

147. A. M. Cuitino and M. Ortiz, "Computational modelling of single crystals," Modelling and Simulation in Materials Science and Engineering, vol. 1, no. 3, p. 225, 1993.

148. S. Harren and R. Asaro, "Nonuniform deformations in polycrystals and aspects of the validity of the taylor model," Journal of the Mechanics and Physics of Solids, vol. 37, no. 2, pp. $191-232,1989$.

149. F. Delaire, J. Raphanel, and C. Rey, "Plastic heterogeneities of a copper multicrystal deformed in uniaxial tension: experimental study and finite element simulations," Acta Materialia, vol. 48, no. 5, pp. 1075 - 1087, 2000.

150. K. K. Mathur and P. R. Dawson, "On modeling the development of crystallographic texture in bulk forming processes," International Journal of Plasticity, vol. 5, no. 1, pp. $67-94,1989$.

151. O. Bouaziz and P. Buessler, "Iso-work increment assumption for heterogeneous material behaviour modelling," Advanced Engineering Materials, vol. 6, no. 1-2, pp. 79-83, 2004.

152. P. Dawson, A. Beaudoin, K. Mathur, and G. Sarma, "Finite element modeling of polycristalline solids," Revue Europénne des Éléments, vol. 3, no. 4, pp. 543-571, 1994.

153. G. Sarma, B. Radhakrishnan, and P. Dawson, "Mesoscale modeling of microstructure and texture evolution during deformation processing of metals," Advanced Engineering Materials, vol. 4, no. 7, pp. 509-514, 2002.

154. L. Meric, P. Poubanne, and G. Cailletaud, "Single crystal modeling for structural calculations. i, model presentation," Journal of Engineering Materials and Technology, vol. 113 , no. 1 , pp. $162-170,1991$.

155. R. A. Lebensohn, "N-site modeling of a $3 \mathrm{D}$ viscoplastic polycrystal using fast fourier transform," Acta Materialia, vol. 49, no. 14, pp. 2723 - 2737, 2001.

156. H. Moulinec and P. Suquet, "A numerical method for computing the overall response of nonlinear composites with complex microstructure," Computer Methods in Applied Mechanics and Engineering, vol. 157, no. 12, pp. 69 - 94, 1998.

157. L. Delannay, S. Kalidindi, and P. V. Houtte, "Quantitative prediction of textures in aluminium cold rolled to moderate strains," Materials Science and Engineering: A, vol. 336, no. 1-2, pp. $233-244,2002$.

158. P. V. Houtte, S. Li, M. Seefeldt, and L. Delannay, "Deformation texture prediction: from the taylor model to the advanced lamel model," International Journal of Plasticity, vol. 21, no. 3, pp. 589 - 624, 2005.

159. M. Zhang, J. Zhang, and D. McDowell, "Microstructure-based crystal plasticity modeling of cyclic deformation of ti6al4v," International Journal of Plasticity, vol. 23, no. 8, pp. $1328-1348,2007$.

160. S. Quilici and G. Cailletaud, "FE simulation of macro-, meso- and micro-scales in 
polycrystalline plasticity," Computational Materials Science, vol. 16, no. 14, pp. 383 - 390, 1999.

161. C. Fahrat and F.-X. Roux, "Implicit Parallel Processing in Structural Mechanics," Computational Mechanics Advances, vol. 2, no. 1, 1994.

162. S.-H. Choi, "Simulation of stored energy and orientation gradients in cold-rolled interstitial free steels," Acta Materialia, vol. 51, no. 6, pp. 1775 - 1788, 2003.

163. G. Sarma and B. Radhakrishnan, "Modeling microstructural effects on the evolution of cube texture during hot deformation of aluminum," Materials Science and Engineering: A, vol. 385, no. 12, pp. $91-104,2004$.

164. E. Héripré, M. Dexet, J. Crépin, L. Gélébart, A. Roos, M. Bornert, and D. Caldemaison, "Coupling between experimental measurements and polycrystal finite element calculations for micromechanical study of metallic materials," International Journal of Plasticity, vol. 23, no. 9, pp. 1512 - 1539, 2007.

165. Z. Zhao, M. Ramesh, D. Raabe, A. Cuitio, and R. Radovitzky, "Investigation of three-dimensional aspects of grain-scale plastic surface deformation of an aluminum oligocrystal," International Journal of Plasticity, vol. 24, no. 12, pp. 2278 - 2297, 2008.

166. F. Barbe, R. Quey, A. Musienko, and G. Cailletaud, "Three-dimensional characterization of strain localization bands in high-resolution elastoplastic polycrystals," Mechanics Research Communications, vol. 36, no. 7, pp. 762 - 768, 2009.

167. Y. Schneider, A. Bertram, T. Böhlke, and C. Hartig, "Plastic deformation behaviour of $\mathrm{Fe}-\mathrm{Cu}$ composites predicted by $3 \mathrm{D}$ finite element simulations," Computational Materials Science, vol. 48, no. 3, pp. 456 - 465, 2010.

168. Y. Schneider, A. Bertram, and T. Böhlke, "Three-dimensional simulation of local and global behaviour of $\alpha \mathrm{Fe}-\mathrm{Cu}$ composites under large plastic deformation," Technische Mechanik, vol. 33, no. 1, pp. 34-51, 2013.

169. L. Li, L. Shen, G. Proust, C. K. Moy, and G. Ranzi, "Three-dimensional crystal plasticity finite element simulation of nanoindentation on aluminium alloy 2024," Materials Science and Engineering: A, vol. 579, no. 0, pp. $41-49,2013$.

170. N. M. Ghoniem, E. P. Busso, N. Kioussis, and H. Huang, "Multiscale modelling of nanomechanics and micromechanics: an overview," Philosophical Magazine, vol. 83, no. 31-34, pp. 3475-3528, 2003.

171. S. Hao, B. Moran, W. Kam Liu, and G. Olson, "A hierarchical multi-physics model for design of high toughness steels," Journal of Computer-Aided Materials Design, vol. 10, no. 2, pp. 99-142, 2003.

172. W. Liu, E. Karpov, S. Zhang, and H. Park, "An introduction to computational nanomechanics and materials," Computer Methods in Applied Mechanics and Engineering, vol. 193, no. 1720, pp. 1529 - 1578, 2004. Multiple Scale Methods for Nanoscale Mechanics and Materials.

173. T. E. Buchheit, G. W. Wellman, and C. C. Battaile, "Investigating the limits of polycrystal plasticity modeling," International Journal of Plasticity, vol. 21, no. 2, pp. $221-249,2005$.

174. T. Bieler, P. Eisenlohr, F. Roters, D. Kumar, D. Mason, M. Crimp, and D. Raabe, "The role of heterogeneous deformation on damage nucleation at grain boundaries in single phase metals," International Journal of Plasticity, vol. 25, no. 9, pp. 1655 - 1683, 2009.

175. D. Raabe, M. Sachtleber, Z. Zhao, F. Roters, and S. Zaefferer, "Micromechanical and macromechanical effects in grain scale polycrystal plasticity experimentation and simulation," Acta Materialia, vol. 49, no. 17, pp. 3433 - 3441, 2001.

176. L. Lim and T. Watanabe, "Fracture toughness and brittle-ductile transition con- 
trolled by grain boundary character distribution (GBCD) in polycrystals," Acta Metallurgica et Materialia, vol. 38, no. 12, pp. 2507 - 2516, 1990.

177. T. Watanabe, "The impact of grain boundary character distribution on fracture in polycrystals," Materials Science and Engineering: A, vol. 176, no. 12, pp. 39 - 49, 1994.

178. G. Palumbo, E. Lehockey, and P. Lin, "Applications for grain boundary engineered materials," JOM, vol. 50, no. 2, pp. 40-43, 1998.

179. C. A. Schuh, M. Kumar, and W. E. King, "Analysis of grain boundary networks and their evolution during grain boundary engineering," Acta Materialia, vol. 51, no. 3, pp. $687-700,2003$

180. V. Randle, "Twinning-related grain boundary engineering," Acta Materialia, vol. 52, no. 14 , pp. $4067-4081,2004$.

181. T. Watanabe and S. Tsurekawa, "Toughening of brittle materials by grain boundary engineering," Materials Science and Engineering: A, vol. 387389, no. 0, pp. 447 455, 2004. 13th International Conference on the Strength of Materials.

182. E. S. McGarrity, P. M. Duxbury, and E. A. Holm, "Statistical physics of grainboundary engineering," Phys. Rev. E, vol. 71, p. 026102, Feb 2005.

183. A. Needleman, "A continuum model for void nucleation by inclusion debonding," Journal of applied mechanics, vol. 54, no. 3, pp. 525-531, 1987.

184. X.-P. Xu and A. Needleman, "Void nucleation by inclusion debonding in a crystal matrix," Modelling and Simulation in Materials Science and Engineering, vol. 1, no. 2, pp. 111-132, 1993.

185. X.-P. Xu and A. Needleman, "Numerical simulations of fast crack growth in brittle solids," Journal of the Mechanics and Physics of Solids, vol. 42, no. 9, pp. 1397 1434, 1994.

186. G. Camacho and M. Ortiz, "Computational modelling of impact damage in brittles materials," International Journal of Solids $\&$ Structures, vol. 33, no. 20-22, pp. 28992938, 1996.

187. M. Ortiz and A. Pandolfi, "Finite-deformation irreversible cohesive elements for three-dimensional crack-propagation analysis," International Journal for Numerical Methods in Engineering, vol. 44, pp. 1267-1282, 1999.

188. Y. Wei and L. Anand, "Grain-boundary sliding and separation in polycrystalline metals: application to nanocrystalline fcc metals," Journal of the Mechanics and Physics of Solids, vol. 52, pp. 2587-2616, 2004.

189. M. Frary and C. A. Schuh, "Connectivity and percolation behaviour of grain boundary networks in three dimensions," Philosophical Magazine, vol. 85, no. 11, pp. 11231143, 2005.

190. M. Kamaya and M. Itakura, "Simulation for intergranular stress corrosion cracking based on a three-dimensional polycrystalline model," Engineering Fracture Mechanics, vol. 76, no. 3, pp. 386 - 401, 2009.

191. L. Meric and G. Cailletaud, "Single crystal modeling for structural calculations. i, model presentation," Journal of Engineering Materials and Technology, vol. 113, no. 1 , pp. 171-182, 1991.

192. G. Cailletaud, "A micromechanical approach to inelastic behaviour of metals," International Journal of Plasticity, vol. 8, no. 1, pp. 55 - 73, 1992.

193. A. Manonukul and F. P. E. Dunne, "High and lowcycle fatigue crack initiation using polycrystal plasticity," Proceedings of the Royal Society of London. Series A: Mathematical, Physical and Engineering Sciences, vol. 460, no. 2047, pp. 1881-1903, 2004.

194. F. Dunne, A. Wilkinson, and R. Allen, "Experimental and computational studies of 
low cycle fatigue crack nucleation in a polycrystal," International Journal of Plasticity, vol. 23, no. 2, pp. 273 - 295, 2007.

195. S. Sinha and S. Ghosh, "Modeling cyclic ratcheting based fatigue life of HSLA steels using crystal plasticity FEM simulations and experiments," International Journal of Fatigue, vol. 28, no. 12, pp. 1690 - 1704, 2006.

196. R. Morrissey, C.-H. Goh, and D. L. McDowell, "Microstructure-scale modeling of HCF deformation," Mechanics of Materials, vol. 35, no. 3-6, pp. 295 - 311, 2003.

197. C. Xie, S. Ghosh, and M. Groeber, "Modeling cyclic deformation of hsla steels using crystal plasticity," Journal of engineering materials and technology, vol. 126, no. 4, pp. 339-352, 2004.

198. A. Ma, F. Roters, and D. Raabe, "On the consideration of interactions between dislocations and grain boundaries in crystal plasticity finite element modeling - theory, experiments, and simulations," Acta Materialia, vol. 54, no. 8, pp. $2181-2194,2006$.

199. J. Querin, J. Schneider, and M. Horstemeyer, "Analysis of micro void formation at grain boundary triple points in monotonically strained AA6022-T43 sheet metal," Materials Science and Engineering: A, vol. 463, no. 1-2, pp. 101 - 106, 2007. TMS 2006, Mukherjee Symposium.

200. R. A. Lebensohn, J. P. Escobedo, E. K. Cerreta, D. Dennis-Koller, C. A. Bronkhorst, and J. F. Bingert, "Modeling void growth in polycrystalline materials," Acta Materialia, vol. 61 , no. 18 , pp. $6918-6932,2013$.

201. M. Vöse, F. Otto, B. Fedelich, and G. Eggeler, "Micromechanical investigations and modelling of a copper - antimony - alloy under creep conditions," Mechanics of Materials, vol. 69, no. 1, pp. $41-62,2014$.

202. T.-S. Han and P. R. Dawson, "A two-scale deformation model for polycrystalline solids using a strongly-coupled finite element methodology," Computer Methods in Applied Mechanics and Engineering, vol. 196, no. 1316, pp. 2029 - 2043, 2007.

203. H. Kuramae, Y. Ikeya, H. Sakamoto, H. Morimoto, and E. Nakamachi, "Multi-scale parallel finite element analyses of LDH sheet formability tests based on crystallographic homogenization method," International Journal of Mechanical Sciences, vol. 52, no. 2, pp. $183-197,2010$.

204. J. Rimoli and M. Ortiz, "A three-dimensional multiscale model of intergranular hydrogen-assisted cracking," Philosophical Magazine, vol. 90, no. 21, pp. 2939-2963, 2010.

205. G. R. Canova, H. R. Wenk, and A. Molinari, "Deformation modelling of multi-phase polycrystals: case of quartz-mica aggregate," Acta Metallurgica et Materialia, vol. 40, pp. 1519-1530, 1992.

206. A. Jean, F. Willot, S. Cantournet, S. Forest, and D. Jeulin, "Large-scale computations of effective elastic properties of rubber with carbon black fillers," International Journal for Multiscale Computational Engineering, vol. 9, pp. 271-303, 2011.

207. A. Burteau, F. NGuyen, J. Bartout, S. Forest, Y. Bienvenu, S. Saberi, and D. Naumann, "Impact of material processing and deformation on cell morphology and mechanical behavior of polyurethane and nickel foams," International Journal of Solids and Structures, vol. 49, pp. 2714-2732, 2012.

208. Neper, Neper: polycrystal generation and meshing (version 2.0). http://neper.sourceforge.net, 2013.

209. S. Forest, "Generalized continuum modelling of crystal plasticity," Generalized continua and dislocation theory, pp. 181-287, Springer, 2012.

210. K. Ammar, B. Appolaire, G. Cailletaud, F. Feyel, and S. Forest, "Finite element formulation of a phase field model based on the concept of generalized stresses," Comput Mat Sc, vol. 45, pp. 800-805, 2009. 
211. A. Gaubert, Y. Le Bouar, and A. Finel, "Coupling phase field and visco-plasticity to study rafting in ni-base superalloys," vol. 90, no. 1, pp. 375-404, 2010.

212. M. Cottura, Y. L. Bouar, A. Finel, B. Appolaire, K. Ammar, and S. Forest, "A phase field model incorporating strain gradient viscoplasticity: Application to rafting in Nibase superalloys," Journal of the Mechanics and Physics of Solids, vol. 60, pp. 1243$1256,2012$. 